\title{
CERTAIN COMBINATORIC CONVOLUTION SUMS AND THEIR RELATIONS TO BERNOULLI AND EULER POLYNOMIALS
}

\author{
Daeyeoul Kim, Abdelmejid Bayad, and Nazli Yildiz Ikikardes
}

\begin{abstract}
In this paper, we give relationship between Bernoulli-Euler polynomials and convolution sums of divisor functions. First, we establish two explicit formulas for certain combinatoric convolution sums of divisor functions derived from Bernoulli and Euler polynomials. Second, as applications, we show five identities concerning the third and fourth-order convolution sums of divisor functions expressed by their divisor functions and linear combination of Bernoulli or Euler polynomials.
\end{abstract}

\section{Introduction and statement of main results}

\subsection{Introduction and notations}

The Bernoulli polynomials $B_{k}(x)$ and the Euler polynomials $E_{k}(x)$ play an important and quite mysterious role in mathematics and various places like analysis, number theory and differential topology. Throughout this paper, the symbols $\mathbb{N}, \mathbb{Z}, \mathbb{R}$ and $\mathbb{C}$ denote the set of natural numbers, the ring of integers, the field of real numbers and the field of complex numbers respectively. For the rest of this subsection we refer to $[6,21]$. The Bernoulli numbers $B_{0}, B_{1}, B_{2}, \ldots$ are defined through the recursion formula

$$
\sum_{j=0}^{k}\left(\begin{array}{c}
k+1 \\
j
\end{array}\right) B_{j}=0 \quad \text { with } B_{0}=1
$$

Received August 18, 2014; Revised November 26, 2014.

2010 Mathematics Subject Classification. 11B68, 11A05, 11K65.

Key words and phrases. Bernoulli polynomials, Euler polynomials, convolution sums, divisor functions.

This work was supported by The Research Fund of Balikesir University, Project No: 2014/32 and by the National Institute for Mathematical Sciences (NIMS) grant funded by the Korean government (B21503-2). 
The Euler numbers $E_{0}, E_{1}, E_{2}, \ldots$ are defined by $E_{0}=1$ and the recursion relation

$$
\sum_{\substack{j=0 \\
2 \mid k-j}}^{k}\left(\begin{array}{l}
k \\
j
\end{array}\right) E_{j}=0 .
$$

For $k \in\{0,1,2, \ldots$,$\} , the k$ th Bernoulli polynomial $B_{k}(x)$ and the $k$ th Euler polynomial $E_{k}(x)$ are defined as follows:

$$
B_{k}(x)=\sum_{j=0}^{k}\left(\begin{array}{l}
k \\
j
\end{array}\right) B_{j} x^{k-j}
$$

and

$$
E_{k}(x)=\sum_{j=0}^{k}\left(\begin{array}{l}
k \\
j
\end{array}\right) \frac{E_{j}}{2^{j}}\left(x-\frac{1}{2}\right)^{k-j} .
$$

Note that both $B_{k}(x)$ and $E_{k}(x)$ are monic polynomials with rational coefficients, $B_{k}=B_{k}(0)$ and $E_{k}=2^{k} E_{k}\left(\frac{1}{2}\right)$. The Bernoulli polynomials and Euler polynomials satisfy the following well-known identities:

$$
\begin{aligned}
& \sum_{j=0}^{N} j^{n}= \frac{B_{n+1}(N+1)-B_{n+1}(0)}{n+1} \\
&= \frac{1}{n+1} \sum_{j=0}^{n}(-1)^{j}\left(\begin{array}{c}
n+1 \\
j
\end{array}\right) B_{j} N^{n+1-j}, \quad(n \geq 1) \\
& B_{n}(x+y)=\sum_{k=0}^{n}\left(\begin{array}{l}
n \\
k
\end{array}\right) B_{k}(x) y^{n-k}
\end{aligned}
$$

and

$$
E_{n}(x+y)=\sum_{k=0}^{n}\left(\begin{array}{l}
n \\
k
\end{array}\right) E_{k}(x) y^{n-k} .
$$

Finally, we need the following number-theoretical functions. For $n \in \mathbb{N}, k \in \mathbb{Z}$ and $l \in\{0,1\}$, we define the following divisor functions:

$$
\begin{aligned}
& \sigma_{k}(n):=\sum_{d \mid n} d^{k}, \quad \sigma_{k}^{*}(n):=\sum_{\substack{d \mid n \\
\frac{n}{d} \text { odd }}} d^{k}, \\
& \sigma_{k, l}(n ; 2):=\sum_{\substack{d \mid n \\
d \equiv l(\bmod 2)}} d^{k}, \quad \widetilde{\sigma}_{k}(n):=\sum_{d \mid n}(-1)^{d-1} d^{k} .
\end{aligned}
$$




\subsection{Motivation}

The identity

$$
\sum_{k=1}^{n-1} \sigma_{1}(k) \sigma_{1}(n-k)=\frac{5}{12} \sigma_{3}(n)+\left(\frac{1}{12}-\frac{1}{2} n\right) \sigma_{1}(n)
$$

for the basic convolution sum first appeared in a letter from Besge to Liouville in 1862 ([3]). Moreover, it should be noted that Ramanujan [18] introduced

$$
\begin{aligned}
& P(q)=1-24 \sum_{n=1}^{\infty} \sigma_{1}(n) q^{n} \\
& Q(q)=1+240 \sum_{n=1}^{\infty} \sigma_{3}(n) q^{n} \\
& R(q)=1-504 \sum_{n=1}^{\infty} \sigma_{5}(n) q^{n}
\end{aligned}
$$

and obtained that

$$
\begin{aligned}
& q \frac{d P(q)}{d q}=\frac{P^{2}(q)-Q(q)}{12}, \\
& q \frac{d Q(q)}{d q}=\frac{P(q) Q(q)-R(q)}{3}, \\
& q \frac{d R(q)}{d q}=\frac{P(q) R(q)-Q^{2}(q)}{2} .
\end{aligned}
$$

Observe that the $n$th coefficient of $P^{2}(q)$ contains the convolution sum

$$
\sum_{k=1}^{n-1} \sigma_{1}(k) \sigma_{1}(n-k)
$$

whence one can see that the identity $q \frac{d P(q)}{d q}=\frac{P^{2}(q)-Q(q)}{12}$ is equivalent to Besge's formula (1.4).

Many recent works on convolution formulas for divisor functions can be found in B. C. Berndt [2], J. W. L. Glaisher [7], H. Hahn [8], J. G. Huard et al. [9], D. Kim et al. [11], G. Melfi [16] and K. S. Williams [25, 24, 26]. In particular, the problem of convolution sums of the divisor function $\sigma_{1}(n)$ and the theory of Eisenstein series has recently attracted considerable interest with the emergence of quasimodular tools. For a similar work, see also [4]. In connection with the classical Jacobi theta and Euler functions, other aspects of the function $\sigma_{1}(n)$ are explored by Simsek in [19]. For some of the story of the subject, and for selection of these articles, we mention [15] and [16], and especially [9] and [25]. The study of convolution sums and their applications is classic and they play an important role in number theory (see [4], [9], [25]). 
In this article we are trying to focus on the combinatoric convolution sums. For positive integers $l$ and $n$, the combinatoric convolution sums

$$
\sum_{r=0}^{l-1}\left(\begin{array}{c}
2 l \\
2 r+1
\end{array}\right) \sum_{m=1}^{n-1} \sigma_{2 l-2 r-1,1}(m ; 2) \sigma_{2 r+1,1}(n-m ; 2)
$$

and

$$
\sum_{s=0}^{k-1}\left(\begin{array}{c}
2 k \\
2 s+1
\end{array}\right) \sum_{m=1}^{n-1} \widetilde{\sigma}_{2 k-2 s-1}(m) \widetilde{\sigma}_{2 s+1}(n-m)
$$

can be evaluated explicitly in terms of divisor functions and a sum involving Bernoulli or Euler polynomials. We are motivated by Ramanujan's recursion formula for sums of the product of two Eisenstein series [2] and its proof, and also the following identities ([10], [25]):

$$
\begin{aligned}
& \sum_{s=0}^{k-1}\left(\begin{array}{c}
2 k \\
2 s+1
\end{array}\right) \sum_{m=1}^{n-1} \sigma_{2 k-2 s-1}(m) \sigma_{2 s+1}(n-m) \\
= & \frac{2 k+3}{4 k+2} \sigma_{2 k+1}(n)+\left(\frac{k}{6}-n\right) \sigma_{2 k-1}(n)+\frac{1}{2 k+1} \sum_{j=2}^{k}\left(\begin{array}{c}
2 k+1 \\
2 j
\end{array}\right) B_{2 j} \sigma_{2 k+1-2 j}(n),
\end{aligned}
$$

$$
\sum_{r=0}^{l-1}\left(\begin{array}{c}
2 l \\
2 r+1
\end{array}\right) \sum_{m=1}^{n-1} \sigma_{2 l-2 r-1}^{*}(m) \sigma_{2 r+1}^{*}(n-m)=\frac{1}{2}\left(\sigma_{2 l+1}^{*}(n)-n \sigma_{2 l-1}^{*}(n)\right) .
$$

\subsection{Main results}

The aim of this article is to study two combinatoric convolution sums of the analogous type (1.5) and (1.6) in [13]. Using these new formulas and addition theorem of Bernoulli or Euler polynomials, we derive the explicit formulas for the third and fourth-order convolution sums of divisor functions.

More precisely, we prove the following results.

Proposition 1. Let $k \geq 1$ and $n \geq 2$. Then

$$
\begin{aligned}
& \sum_{s=0}^{k-1}\left(\begin{array}{c}
2 k \\
2 s+1
\end{array}\right) \sum_{m=1}^{n-1} \sigma_{2 k-2 s-1,1}(m ; 2) \sigma_{2 s+1,1}(n-m ; 2) \\
= & \frac{1}{4} \sigma_{2 k+1,0}(n ; 2)+\frac{2^{2 k}}{2 k+1} \sum_{\substack{d \mid n \\
d \text { odd }}} B_{2 k+1}\left(\frac{d+1}{2}\right) .
\end{aligned}
$$


In the following special cases the above formula in Proposition 1 is simple. Then, for $n$ odd we have

$$
\begin{aligned}
& (2 k+1) \sum_{s=0}^{k-1}\left(\begin{array}{c}
2 k \\
2 s+1
\end{array}\right) \sum_{m=1}^{n-1} \sigma_{2 k-2 s-1,1}(m ; 2) \sigma_{2 s+1,1}(n-m ; 2) \\
= & 2^{2 k} \sum_{d \mid n} B_{2 k+1}\left(\frac{d+1}{2}\right) .
\end{aligned}
$$

If $n=2^{a}$, then

$$
\sum_{s=0}^{k-1}\left(\begin{array}{c}
2 k \\
2 s+1
\end{array}\right) \sum_{m=1}^{n-1} \sigma_{2 k-2 s-1,1}(m ; 2) \sigma_{2 s+1,1}(n-m ; 2)=\frac{1}{4} \sigma_{2 k+1,0}\left(2^{a} ; 2\right) .
$$

We set

$$
\left(\begin{array}{c}
m \\
a_{1}, \ldots, a_{k}
\end{array}\right)=\frac{m !}{a_{1} ! \cdots a_{k} !} \text {, with } a_{1}+\cdots+a_{k}=m \text { and } a_{1}, \ldots, a_{k} \in \mathbb{N} \cup\{0\} \text {. }
$$

Corollary 2. Let $n \geq 1$ and $q$ be an odd positive integer greater than 2 . Then

$$
\begin{aligned}
& \sum_{\substack{1 \leq k \leq n \\
1 \leq t \leq 2 k, t \text { odd }}}\left(\begin{array}{c}
2 n+1+2 k \\
2 k, 2 n+1-2 k, t, 2 k-t
\end{array}\right)\left(\sum_{m=1}^{q-1} \frac{\sigma_{2 k-t, 1}(m ; 2)}{2^{2 k-t}} \frac{\sigma_{t, 1}(q-m ; 2)}{2^{t-1}}\right) \\
= & \sum_{d \mid q} P_{2 n+1}(d)-\sigma_{1}(q),
\end{aligned}
$$

where $P_{n}(x)$ is the Legendre polynomial. If $p$ is an odd positive prime integer, then

$$
\sum_{d \mid q} P_{p}(d) \equiv \sigma_{1}(q) \quad(\bmod p)
$$

In particular, if $p$ and $q$ are distinct odd positive prime integers, then we have

$$
P_{p}(q) / q \equiv 1 \quad(\bmod p) .
$$

Remark 3. (1) Many interesting results of the Legendre polynomial are in $[22,23]$. By $(1.10)$, we give a finding method for a composite number. That is, if $P_{p}(q) / q \not \equiv 1(\bmod p)$, then we determine $q$ is a composite number.

(2) If $p$ is an odd positive prime integer, then

$$
\sum_{s=0}^{k-1}\left(\begin{array}{c}
2 k \\
2 s+1
\end{array}\right) \sum_{m=1}^{p-1}\left(\frac{\sigma_{2 k-2 s-1,1}(m ; 2)}{2^{2 k-2 s-1}}\right)\left(\frac{\sigma_{2 s+1,1}(p-m ; 2)}{2^{2 s+1}}\right)=\sum_{j=0}^{(p-1) / 2} j^{2 k} .
$$

This is an analogous answer of (Question) in [12, p. 336].

Theorem 4. Let $k$ and $l$ be nonnegative integers. Then we have

$$
\sum_{j+r+v=2 k+1} \frac{(-1)^{v}}{2^{r+v}}\left(\begin{array}{c}
2 k+1 \\
j, r, v
\end{array}\right) B_{j} \cdot(2 l+1)^{v}=-B_{2 k+1}(l+1) .
$$


Example 5. If $p=2 q+1$ is an odd prime, then

$$
\begin{aligned}
& \sum_{m=1}^{q} \sigma_{1,1}(m ; 2) \sigma_{1,1}(2 q+1-m ; 2)=\frac{B_{3}(q+1)}{3}, \\
& \sum_{m=1}^{q} \sigma_{1,1}(m ; 2) \sigma_{3,1}(2 q+1-m ; 2)=\frac{B_{5}(q+1)}{5}, \\
& \sum_{j+r+v=2 k+1} \frac{(-1)^{v}}{2^{r+v}}\left(\begin{array}{c}
2 k+1 \\
j, r, v
\end{array}\right) B_{j} \cdot p^{v}=-B_{2 k+1}\left(\frac{p+1}{2}\right) .
\end{aligned}
$$

Proposition 6. Let $k \geq 1$ and $n \geq 2$. Then

$$
\begin{aligned}
& \sum_{s=0}^{k-1}\left(\begin{array}{c}
2 k \\
2 s+1
\end{array}\right) \sum_{m=1}^{n-1} \widetilde{\sigma}_{2 k-2 s-1}(m) \widetilde{\sigma}_{2 s+1}(n-m) \\
= & -\frac{1}{2} \widetilde{\sigma}_{2 k+1}(n)+\frac{1}{2} \widetilde{\sigma}_{2 k}(n)+n \widetilde{\sigma}_{2 k-1}(n)-\frac{1}{2} \sum_{d \mid n}(-1)^{d-1} E_{2 k}(d+1) .
\end{aligned}
$$

Remark 7. (1) (1.15) is a generalization of [8, (4.4), (4.9)].

(2) The Proposition 1 and Proposition 6 can be compare to Theorem 3 and Theorem 4 in [13] as different formulas, respectively. Bernoulli, Euler polynomials and the convolutions for divisor functions are studied by many mathematicians independently. Our results (Propositions 1 and 4) give us two good relations between Bernoulli-Euler polynomials and convolution sum of divisor functions. For example, the well known basic properties (addition, difference, symmetry formulas, ..., etc) of Bernoulli and Euler polynomials give us a simple and useful computational technique for the third and fourthorder convolution sum of divisor functions. In particular, it is a curious result with respect to same pattern that a certain combinatoric of convolution sums for $\left(\sum_{d \mid n}(-1)^{d-1} d^{2 k-2 s-1}\right)\left(\sum_{d \mid n}(-1)^{d-1} d^{2 s+1}\right)$ in Proposition 6 is represented by a linear sum with respect to a Euler sum $\sum_{d \mid n}(-1)^{d-1} E_{2 k}(d+1)$ and three divisor functions $\sum_{d \mid n}(-1)^{d-1} d^{2 k+1-i}$ with $i=0,1,2$.

As applications of Proposition 1 and Proposition 6, we obtain the following results.

Theorem 8. Let $l, n, p, q \in \mathbb{N}$ with $l, n, p$ greater than 1 . Then

(a)

$$
\begin{aligned}
& \sum_{\substack{a, b \text { odd } \\
1 \leq m \leq n-1}}\left(\begin{array}{c}
l \\
a, b, c
\end{array}\right) \sigma_{a, 1}(m ; 2) \sigma_{b, 1}(n-m ; 2) \sigma_{c, 0}(2 q ; 2) \\
= & \frac{2^{l-2}}{l+1} \sum_{\alpha\left|\frac{n}{2}, d\right| q} \alpha\left[B_{l+1}(\alpha+d+1)-B_{l+1}(\alpha+d)\right. \\
& \left.+(-1)^{l}\left\{B_{l+1}(\alpha-d+1)-B_{l+1}(\alpha-d)\right\}\right] \\
+ & \frac{2^{l-1}}{l+1}\left[\sum_{\substack{d|q \\
\alpha| n, \alpha \text { odd }}}\left(B_{l+1}\left(\frac{\alpha+1}{2}+d\right)+(-1)^{l} B_{l+1}\left(\frac{\alpha+1}{2}-d\right)\right)\right]
\end{aligned}
$$




$$
-\frac{1}{2}\left(\sigma_{1,1}(n ; 2)+\sigma_{1}\left(\frac{n}{2}\right)\right) \sigma_{l, 0}(2 q ; 2)
$$

(b)

$$
\begin{aligned}
& \sum_{\substack{a, b \text { odd } \\
1 \leq m \leq n-1}}\left(\begin{array}{c}
l \\
a, b, c
\end{array}\right) \sigma_{a, 1}(m ; 2) \sigma_{b, 1}(n-m ; 2) \sigma_{c}^{*}(2 q) \\
= & \frac{2^{l-2}}{l+1} \sum_{\substack{\alpha\left|\frac{n}{2} \\
d\right| q, \frac{q}{d} \text { odd }}} \alpha\left\{B_{l+1}(\alpha+d+1)-B_{l+1}(\alpha+d)\right. \\
& \left.+(-1)^{l}\left(B_{l+1}(\alpha-d+1)+B_{l+1}(\alpha-d)\right)\right\} \\
+ & \frac{2^{l-1}}{l+1}\left[\sum_{\substack{d|q \\
\alpha| n, \alpha \text { odd }}}\left(B_{l+1}\left(\frac{\alpha+1}{2}+d\right)+(-1)^{l} B_{l+1}\left(\frac{\alpha+1}{2}-d\right)\right)\right] \\
- & \frac{1}{2}\left(\sigma_{1,1}(n ; 2)+\sigma_{1}\left(\frac{n}{2}\right)\right) \sigma_{l}^{*}(2 q) .
\end{aligned}
$$

(c)

$$
\begin{gathered}
\sum_{\substack{a, b \text { odd } \\
1 \leq m \leq p-1}}\left(\begin{array}{c}
l \\
a, b, c
\end{array}\right) \widetilde{\sigma}_{a}(m) \widetilde{\sigma}_{b}(p-m) \widetilde{\sigma}_{c}(q) \\
=\frac{1}{2} \widetilde{\sigma}_{l}(q)\left\{\widetilde{\sigma}_{1}(p)-2 p \widetilde{\sigma}_{-1}(p)\right\} \\
\quad+\frac{1}{8} \sum_{\alpha|p, d| q} \frac{(-1)^{\alpha+d}}{\alpha}\left[\left(2 p+\alpha-\alpha^{2}\right)\left\{E_{l}(d+\alpha)+E_{l}(d-\alpha+1)\right\}\right. \\
\left.\quad+\left(2 p-\alpha-\alpha^{2}\right)\left\{E_{l}(d+\alpha+1)+E_{l}(d-\alpha)\right\}\right] .
\end{gathered}
$$

Theorem 9. Let $m, p, q \in \mathbb{N}$ with greater than 1 . Then

$$
\begin{gathered}
\sum_{\substack{1 \leq l \leq p-1 \\
1 \leq l^{\prime} \leq q-1 \\
a, b, c, d \text { odd }}}\left(\begin{array}{c}
2 m \\
a, b, c, d
\end{array}\right) \sigma_{a, 1}(l ; 2) \sigma_{b, 1}(p-l ; 2) \sigma_{c, 1}\left(l^{\prime} ; 2\right) \sigma_{d, 1}\left(q-l^{\prime} ; 2\right) \\
=\sum_{\substack{d\left|p, d^{\prime}\right| q \\
d, d^{\prime} \text { odd }}}\left[\frac { 2 ^ { 2 m - 2 } } { 2 m + 1 } \left\{\left(d+d^{\prime}\right) B_{2 m+1}\left(\frac{d+d^{\prime}}{2}+1\right)+\left(d-d^{\prime}\right) B_{2 m+1}\left(\frac{d^{\prime}-d}{2}\right)\right.\right. \\
\left.\quad-2 d B_{2 m+1}\left(\frac{d^{\prime}+1}{2}\right)-2 d^{\prime} B_{2 m+1}\left(\frac{d+1}{2}\right)\right\} \\
\left.\quad-\frac{2^{2 m-2}}{m+1}\left\{B_{2 m+2}\left(\frac{d+d^{\prime}}{2}+1\right)-B_{2 m+2}\left(\frac{d^{\prime}-d}{2}\right)\right\}\right]
\end{gathered}
$$




$$
\begin{aligned}
& +\frac{2^{2 m-2}}{2 m+1} \sum_{\substack{d\left|\frac{p}{2}, d^{\prime}\right| q \\
d^{\prime} \text { odd }}} d\left[B_{2 m+1}\left(\frac{d^{\prime}+1+2 d}{2}\right)+B_{2 m+1}\left(\frac{d^{\prime}+1-2 d}{2}\right)\right] \\
& +\frac{2^{2 m-2}}{2 m+1} \sum_{\substack{d^{\prime}\left|\frac{q}{2}, d\right| p \\
d \text { odd }}} d^{\prime}\left[B_{2 m+1}\left(\frac{d+1+2 d^{\prime}}{2}\right)+B_{2 m+1}\left(\frac{d+1-2 d^{\prime}}{2}\right)\right] \\
& +\frac{2^{2 m-3}}{2 m+1} \sum_{d\left|\frac{p}{2}, d^{\prime}\right| \frac{q}{2}} d d^{\prime}\left\{B_{2 m+1}\left(d+d^{\prime}+1\right)-B_{2 m+1}\left(d+d^{\prime}\right)\right. \\
& \left.+B_{2 m+1}\left(d-d^{\prime}+1\right)-B_{2 m+1}\left(d-d^{\prime}\right)\right\} \\
& -\frac{2^{2 m-2}}{2 m+1}\left\{\sigma_{1,0}(p ; 2) \sum_{d^{\prime} \mid q, d^{\prime} \text { odd }} B_{2 m+1}\left(\frac{d^{\prime}+1}{2}\right)\right. \\
& \left.+\sigma_{1,0}(q ; 2) \sum_{d \mid p, d \text { odd }} B_{2 m+1}\left(\frac{d+1}{2}\right)\right\} \\
& -\frac{1}{8}\left\{\sigma_{2 m+1,0}(q ; 2) \sigma_{1,1}(p ; 2)+\sigma_{2 m+1,0}(p ; 2) \sigma_{1,1}(q ; 2)\right\} \\
& -\frac{1}{16}\left\{\sigma_{2 m+1,0}(p ; 2) \sigma_{1,0}(q ; 2)+\sigma_{1,0}(p ; 2) \sigma_{2 m+1,0}(q ; 2)\right\} \text {. }
\end{aligned}
$$

Example 10. Theorem 9 in the special case where $p$ and $q$ are odd positive prime integers, has the following nice and simple statement:

$$
\begin{gathered}
\sum_{\substack{1 \leq l \leq p-1,1 \leq l^{\prime} \leq q-1 \\
a, b, c, d}}\left(\begin{array}{c}
2 m \\
\text { odd } \\
a, b, c, d
\end{array}\right) \sigma_{a, 1}(l ; 2) \sigma_{b, 1}(p-l ; 2) \sigma_{c, 1}\left(l^{\prime} ; 2\right) \sigma_{d, 1}\left(q-l^{\prime} ; 2\right) \\
=\frac{4^{m-1}}{2 m+1}\left\{(p+q) B_{2 m+1}\left(\frac{p+q}{2}+1\right)+(p-q) B_{2 m+1}\left(\frac{q-p}{2}\right)\right. \\
\left.-2 p B_{2 m+1}\left(\frac{q+1}{2}\right)-2 q B_{2 m+1}\left(\frac{p+1}{2}\right)\right\} \\
-\frac{4^{m-1}}{m+1}\left\{B_{2 m+2}\left(\frac{p+q}{2}+1\right)-B_{2 m+2}\left(\frac{q-p}{2}\right)\right\} .
\end{gathered}
$$

Theorem 11. Let $m, p, q \in \mathbb{N}$ with greater than 1 . Then the triple convolution sum

$$
\begin{gathered}
\sum_{\substack{1 \leq l \leq p-1,1 \leq l^{\prime} \leq q-1 \\
a, b, c, d \text { odd }}}\left(\begin{array}{c}
2 m \\
a, b, c, d
\end{array}\right) \widetilde{\sigma}_{a}(l) \widetilde{\sigma}_{b}(p-l) \widetilde{\sigma}_{c}\left(l^{\prime}\right) \widetilde{\sigma}_{d}\left(q-l^{\prime}\right) \\
=-\frac{1}{4}\left(\widetilde{\sigma}_{1}(p)-2 p \widetilde{\sigma}_{-1}(p)\right)\left(\widetilde{\sigma}_{2 m+1}(q)-\widetilde{\sigma}_{2 m}(q)-2 q \widetilde{\sigma}_{2 m-1}(q)\right.
\end{gathered}
$$




$$
\begin{gathered}
\left.+\sum_{d^{\prime} \mid q}(-1)^{d^{\prime}-1} E_{2 m}\left(d^{\prime}+1\right)\right) \\
-\frac{1}{4}\left(\widetilde{\sigma}_{1}(q)-2 q \widetilde{\sigma}_{-1}(q)\right)\left(\widetilde{\sigma}_{2 m+1}(p)-\widetilde{\sigma}_{2 m}(p)-2 p \widetilde{\sigma}_{2 m-1}(p)\right. \\
\left.+\sum_{d \mid p}(-1)^{d-1} E_{2 m}(d+1)\right) \\
+\frac{1}{8} \sum_{d\left|p, d^{\prime}\right| q}(-1)^{d+d^{\prime}}\left[\frac{\left(2 p+d-d^{2}\right)\left(2 q+d^{\prime}-d^{\prime 2}\right)}{2 d d^{\prime}}\right. \\
\times\left\{E_{2 m}\left(d+d^{\prime}+1\right)+E_{2 m}\left(d+d^{\prime}\right)+E_{2 m}\left(d-d^{\prime}\right)+E_{2 m}\left(d-d^{\prime}+1\right)\right\} \\
-\left\{\frac{\left(2 p+d-d^{2}\right)}{d}+\frac{\left(2 q+d^{\prime}-d^{\prime 2}\right)}{d^{\prime}}\right\} E_{2 m}\left(d+d^{\prime}+1\right) \\
-\frac{\left(2 p+d\left(1+2 d^{\prime}\right)-3 d^{2}\right)}{d} E_{2 m}\left(d-d^{\prime}\right)-\frac{\left(2 q+d^{\prime}-d^{\prime 2}\right)}{d^{\prime}} E_{2 m}\left(d-d^{\prime}+1\right) \\
+2\left\{E_{2 m+1}\left(d+d^{\prime}+2\right)+E_{2 m+1}\left(d^{\prime}-d+1\right)\right. \\
\left.\left.-\left(d+d^{\prime}+1\right) E_{2 m}\left(d+d^{\prime}+2\right)\right\}\right] .
\end{gathered}
$$

Observe that in the case $p$ and $q$ odd prime numbers, the above formula is very simple.

\section{Proofs of first main results}

To prove propositions and theorems, we need the following lemma.

Lemma 12. If $n \geq 2$ and $k \geq 1$, then

$$
\begin{aligned}
& \sum_{s=0}^{k-1}\left(\begin{array}{c}
2 k \\
2 s+1
\end{array}\right) \sum_{m=1}^{n-1} \sigma_{2 k-2 s-1}(m) \sigma_{2 s+1}(n-m) \\
= & \frac{1}{2} \sigma_{2 k+1}(n)-\frac{1}{2} \sigma_{2 k}(n)-n \sigma_{2 k-1}(n)+\sum_{d \mid n} \frac{B_{2 k+1}(d+1)}{2 k+1} .
\end{aligned}
$$

Proof. From (1.7) we see that

$$
\begin{aligned}
& \sum_{s=0}^{k-1}\left(\begin{array}{c}
2 k \\
2 s+1
\end{array}\right) \sum_{m=1}^{n-1} \sigma_{2 k-2 s-1}(m) \sigma_{2 s+1}(n-m) \\
= & \frac{2 k+3}{4 k+2} \sigma_{2 k+1}(n)+\left(\frac{k}{6}-n\right) \sigma_{2 k-1}(n)+\frac{1}{2 k+1} \sum_{j=2}^{k}\left(\begin{array}{c}
2 k+1 \\
2 j
\end{array}\right) B_{2 j} \sigma_{2 k+1-2 j}(n) \\
= & \frac{2 k+3}{4 k+2} \sigma_{2 k+1}(n)+\left(\frac{k}{6}-n\right) \sigma_{2 k-1}(n)+\frac{1}{2 k+1} \sum_{j=0}^{2 k+1}\left(\begin{array}{c}
2 k+1 \\
j
\end{array}\right)(-1)^{j} B_{j} \sigma_{2 k+1-j}(n)
\end{aligned}
$$




$$
\begin{gathered}
-\frac{1}{2 k+1}\left(\sum_{j=0}^{k}\left(\begin{array}{c}
2 k+1 \\
2 j+1
\end{array}\right)(-1)^{2 j+1} B_{2 j+1} \sigma_{2 k-2 j}(n)+B_{0} \sigma_{2 k+1}(n)\right. \\
\left.+\left(\begin{array}{c}
2 k+1 \\
2
\end{array}\right) B_{2} \sigma_{2 k-1}(n)\right) .
\end{gathered}
$$

Using relation (1.1) and

$$
B_{0}=1, B_{1}=-\frac{1}{2}, B_{2}=\frac{1}{6}, B_{2 k+1}=B_{2 k+1}(0)=0, \text { with } k \geq 1
$$

we deduce that

$$
\begin{aligned}
& \sum_{j=0}^{k}\left(\begin{array}{c}
2 k+1 \\
2 j+1
\end{array}\right)(-1)^{2 j+1} B_{2 j+1} \sigma_{2 k-2 j}(n) \\
= & -\left(\begin{array}{c}
2 k+1 \\
1
\end{array}\right) B_{1} \sigma_{2 k}(n)=\frac{2 k+1}{2} \sigma_{2 k}(n), \\
& \frac{1}{2 k+1} \sum_{j=0}^{2 k+1}\left(\begin{array}{c}
2 k+1 \\
j
\end{array}\right)(-1)^{j} B_{j} \sigma_{2 k+1-j}(n) \\
= & \sum_{d \mid n} \frac{1}{2 k+1} \sum_{j=0}^{2 k+1}\left(\begin{array}{c}
2 k+1 \\
j
\end{array}\right)(-1)^{j} B_{j} d^{2 k+1-j} \\
= & \sum_{d \mid n} \frac{B_{2 k+1}(d+1)-B_{2 k+1}(0)}{2 k+1}=\frac{1}{2 k+1} \sum_{d \mid n} B_{2 k+1}(d+1)
\end{aligned}
$$

with $k \geq 1$. Combining (2.1), (2.2) and (2.3), we get this lemma.

Remark 13. The lemma gives new light, to study higher combinatoric convolution sums. In fact, we will use it to prove Proposition 6 .

In the following proposition, we state a property of combinatoric convolution sums for divisor functions, which will be used in our proofs.

Proposition $14([13,(12)])$. For $k, n \in \mathbb{N}$ and $n \geq 2$,

$$
\begin{aligned}
& \sum_{s=0}^{k-1}\left(\begin{array}{c}
2 k \\
2 s+1
\end{array}\right) \sum_{m=1}^{n-1} \sigma_{2 k-2 s-1,1}(m ; 2) \sigma_{2 s+1,1}(n-m ; 2) \\
= & 2^{2 k-1} \sigma_{2 k+1}\left(\frac{n}{2}\right)+\frac{1}{4 k+2} \sum_{i=0}^{k}\left(\begin{array}{c}
2 k+1 \\
2 i+1
\end{array}\right) \tilde{B}_{2 k-2 i} \sigma_{2 i+1,1}(n ; 2),
\end{aligned}
$$

where $\widetilde{B}_{k}:=\sum_{j=0}^{k}\left(\begin{array}{c}k \\ j\end{array}\right) 2^{j} B_{j}=2^{k} B_{k}(1 / 2)$.

Proof of Proposition 1. By (1.2) we obtain we obtain

$$
B_{2 k+1}\left(\frac{1+d}{2}\right)-B_{2 k+1}\left(\frac{1-d}{2}\right)=\frac{1}{2^{2 k}} \sum_{i=0}^{k}\left(\begin{array}{c}
2 k+1 \\
2 i
\end{array}\right) B_{2 i}\left(\frac{1}{2}\right) 2^{2 i} d^{2 k+1-2 i}
$$


We recall that

$$
B_{n}(1-x)=(-1)^{n} B_{n}(x) .
$$

Using (2.4) and (2.5), we deduce that

$$
B_{2 k+1}\left(\frac{d+1}{2}\right)=\frac{1}{2^{2 k+1}} \sum_{i=0}^{k}\left(\begin{array}{c}
2 k+1 \\
2 i+1
\end{array}\right) \tilde{B}_{2 k-2 i} d^{2 i+1} .
$$

Equating (2.6) and Proposition 14,

$$
\begin{aligned}
& \sum_{s=0}^{k-1}\left(\begin{array}{c}
2 k \\
2 s+1
\end{array}\right) \sum_{m=1}^{n-1} \sigma_{2 k-2 s-1,1}(m ; 2) \sigma_{2 s+1,1}(n-m ; 2) \\
= & \frac{1}{4} \sigma_{2 k+1,0}(n ; 2)+\frac{2^{2 k}}{2 k+1} \sum_{\substack{d \mid n \\
d \text { odd }}} B_{2 k+1}\left(\frac{d+1}{2}\right)
\end{aligned}
$$

with $k \geq 1$. It is easily checked that $\sigma_{2 k+1,0}(n ; 2)=0$ with $n$ odd. Thus we obtain

$$
\begin{aligned}
& 2^{2 k} \sum_{\substack{d \mid n \\
d \text { odd }}} B_{2 k+1}\left(\frac{d+1}{2}\right) \\
= & (2 k+1) \sum_{s=0}^{k-1}\left(\begin{array}{c}
2 k \\
2 s+1
\end{array}\right) \sum_{m=1}^{n-1} \sigma_{2 k-2 s-1,1}(m ; 2) \sigma_{2 s+1,1}(n-m ; 2)
\end{aligned}
$$

with $n$ odd. If $n=2^{a}$, then $\sum_{\substack{\left.d\right|^{a} \text { odd } \\ \text { a }}} B_{2 k+1}\left(\frac{d+1}{2}\right)=B_{2 k+1}(1)=0$. This proves the proposition.

Proof of Corollary 2. We recall [17, (2.6)] that

$$
\frac{1}{2} P_{2 n+1}(2 x-1)=\sum_{k=0}^{n} \frac{(2 n+2 k+1) !}{(2 k) !(2 k+1) !(2 n-2 k+1) !} B_{2 k+1}(x)
$$

with $n \in \mathbb{N}$. Put $d=2 x-1$. By (2.7) and (1.9), we derive that

$$
\begin{aligned}
& \sum_{d \mid q} P_{2 n+1}(d)=2 \sum_{d \mid q} \sum_{k=0}^{n} \frac{(2 n+1+2 k) !}{(2 k) !(2 k) !(2 n+1-2 k) !} \frac{B_{2 k+1}\left(\frac{d+1}{2}\right)}{2 k+1} \\
& =\sum_{d \mid q} d+\sum_{k=1}^{n}\left(\begin{array}{c}
2 n+1+2 k \\
2 k, 2 k, 2 n+1-2 k
\end{array}\right) \\
& \times\left(\sum_{s=0}^{k-1}\left(\begin{array}{c}
2 k \\
2 s+1
\end{array}\right) \sum_{m=1}^{q-1} \frac{\sigma_{2 k-2 s-1,1}(m ; 2)}{2^{2 k-2 s-1}} \frac{\sigma_{2 s+1,1}(q-m ; 2)}{2^{2 s}}\right) \\
& =\sigma_{1}(q)+\sum_{\substack{1 \leq k \leq n \\
1 \leq t \leq 2 k, t \text { odd }}}\left(\begin{array}{c}
2 n+1+2 k \\
2 k, 2 n+1-2 k, t, 2 k-t
\end{array}\right)
\end{aligned}
$$




$$
\times\left(\sum_{m=1}^{q-1} \frac{\sigma_{2 k-t, 1}(m ; 2)}{2^{2 k-t}} \frac{\sigma_{2 t, 1}(q-m ; 2)}{2^{t-1}}\right) .
$$

It is easily checked that $P_{p}(1)=1, \sigma_{1}(q)=1+q$ and $p \mid\left(\begin{array}{c}p+2 k \\ 2 k, p-2 k, t, 2 k-t\end{array}\right)$ with $p$ and $q$ distinct odd prime integers and $1 \leq k \leq(p-1) / 2$. This proves the corollary.

Proof of Theorem 4. For $k=0$, by simple computation Theorem 4 holds. By the same method in (2.3), we derive that

$$
\begin{aligned}
& -2 \sum_{\substack{d \mid n \\
d \text { odd }}} \sum_{\substack{1 \leq t<d \\
t \text { even }}} t^{2 k}=-2^{2 k+1} \sum_{\substack{d \mid n \\
d \text { odd }}} \sum_{l=1}^{\frac{d-1}{2}} l^{2 k} \\
= & -2^{2 k+1} \sum_{\substack{d \mid n \\
d \text { odd }}} \frac{1}{2 k+1} \sum_{j=0}^{2 k}(-1)^{j}\left(\begin{array}{c}
2 k+1 \\
j
\end{array}\right) B_{j}\left(\frac{d-1}{2}\right)^{2 k+1-j} \\
= & \sum_{j=0}^{2 k}(-1)^{j+1}\left(\begin{array}{c}
2 k+1 \\
j
\end{array}\right) \frac{2^{j} B_{j}}{2 k+1} \sum_{\substack{d \mid n \\
d \text { odd }}}(d-1)^{2 k+1-j} \\
= & \sum_{j=0}^{2 k}(-1)^{j+1}\left(\begin{array}{c}
2 k+1 \\
j
\end{array}\right) \frac{2^{j} B_{j}}{2 k+1} \sum_{\substack{d|n \\
d| n}}^{2 k+1-j} \sum_{r=0}^{2 k}\left(\begin{array}{c}
2 k+1-j \\
r
\end{array}\right)(-1)^{r} d^{2 k+1-j-r} \\
= & \sum_{j=0}^{2 k}(-1)^{j+1}\left(\begin{array}{c}
2 k+1 \\
j
\end{array}\right) \frac{2^{j} B_{j}}{2 k+1} \sum_{r=0}^{2 k+1-j}\left(\begin{array}{c}
2 k+1-j \\
r
\end{array}\right)(-1)^{r} \sigma_{2 k+1-j-r, 1}(n ; 2) \\
= & \sum_{j=0}^{2 k} \sum_{r=0}^{2 k+1-j}(-1)^{j+r+1}\left(\begin{array}{c}
2 k+1 \\
j
\end{array}\right)\left(\begin{array}{c}
2 k+1-j \\
r
\end{array}\right) \frac{2^{j} B_{j}}{2 k+1} \sigma_{2 k+1-j-r, 1}(n ; 2)
\end{aligned}
$$

with $k \geq 1$. Therefore, we get

$$
\begin{aligned}
& \frac{1}{4} \sigma_{2 k+1,0}(n ; 2)+\frac{2^{2 k}}{2 k+1} \sum_{\substack{d \mid n \\
d \text { odd }}} B_{2 k+1}\left(\frac{d+1}{2}\right) \\
= & \frac{1}{4} \sigma_{2 k+1,0}(n ; 2) \\
& +\frac{1}{4 k+2} \sum_{j=0}^{2 k} \sum_{r=0}^{2 k+1-j}(-1)^{j+r}\left(\begin{array}{c}
2 k+1 \\
j
\end{array}\right)\left(\begin{array}{c}
2 k+1-j \\
r
\end{array}\right) 2^{j} B_{j} \sigma_{2 k+1-j-r, 1}(n ; 2)
\end{aligned}
$$

and

$$
\sum_{2 l+1 \mid n} B_{2 k+1}(l+1)=-\sum_{2 l+1 \mid n}\left(\sum_{j+r+v=2 k+1}(2 l+1)^{v}(-1)^{v}\left(\begin{array}{c}
2 k+1 \\
j, r, v
\end{array}\right) 2^{-r-v} B_{j}\right) .
$$

We get the theorem. 
Remark 15. (1.11) is an analogous result in [11, Question].

Lemma 16. Let $k \geq 1$ and $n \geq 4$. Then we obtain

$$
\begin{aligned}
& \sum_{s=0}^{k-1}\left(\begin{array}{c}
2 k \\
2 s+1
\end{array}\right) \sum_{m=1}^{n-1} \sigma_{2 k-2 s-1}\left(\frac{m}{2}\right) \sigma_{2 s+1}\left(\frac{n-m}{2}\right) \\
= & \frac{1}{2} \sigma_{2 k+1}\left(\frac{n}{2}\right)-\frac{1}{2} \sigma_{2 k}\left(\frac{n}{2}\right)-\frac{n}{2} \sigma_{2 k-1}\left(\frac{n}{2}\right)+\sum_{d \mid \frac{n}{2}} \frac{B_{2 k+1}(d+1)}{2 k+1} .
\end{aligned}
$$

Proof. To prove this lemma, we consider

$$
\begin{aligned}
& \sum_{s=0}^{k-1}\left(\begin{array}{c}
2 k \\
2 s+1
\end{array}\right) \sum_{m=1}^{n-1} \sigma_{2 k-2 s-1}\left(\frac{m}{2}\right) \sigma_{2 s+1}\left(\frac{n-m}{2}\right) \\
= & \sum_{s=0}^{k-1}\left(\begin{array}{c}
2 k \\
2 s+1
\end{array}\right) \sum_{m=1}^{\frac{n}{2}-1} \sigma_{2 k-2 s-1}(m) \sigma_{2 s+1}\left(\frac{n}{2}-m\right) .
\end{aligned}
$$

By Lemma 12, we have

$$
\begin{aligned}
& \sum_{s=0}^{k-1}\left(\begin{array}{c}
2 k \\
2 s+1
\end{array}\right) \sum_{m=1}^{n-1} \sigma_{2 k-2 s-1}\left(\frac{m}{2}\right) \sigma_{2 s+1}\left(\frac{n-m}{2}\right) \\
= & \frac{1}{2} \sigma_{2 k+1}\left(\frac{n}{2}\right)-\frac{1}{2} \sigma_{2 k}\left(\frac{n}{2}\right)-\frac{n}{2} \sigma_{2 k-1}\left(\frac{n}{2}\right)+\sum_{d \mid \frac{n}{2}} \frac{B_{2 k+1}(d+1)}{2 k+1} .
\end{aligned}
$$

Proof of Proposition 6. Let $k \geq 1$ and $n \geq 2$. Then we obtain

$$
\begin{aligned}
& \sum_{s=0}^{k-1}\left(\begin{array}{c}
2 k \\
2 s+1
\end{array}\right) \sum_{m=1}^{n-1} \tilde{\sigma}_{2 k-2 s-1}(m) \widetilde{\sigma}_{2 s+1}(n-m) \\
& =\sum_{s=0}^{k-1}\left(\begin{array}{c}
2 k \\
2 s+1
\end{array}\right) \sum_{m=1}^{n-1}\left(\sigma_{2 k-2 s-1}(m)-2^{2 k-2 s} \sigma_{2 k-2 s-1}\left(\frac{m}{2}\right)\right) \\
& \times\left(\sigma_{2 s+1}(n-m)-2^{2 s+2} \sigma_{2 s+1}\left(\frac{n-m}{2}\right)\right) \\
& =\sum_{s=0}^{k-1}\left(\begin{array}{c}
2 k \\
2 s+1
\end{array}\right) \sum_{m=1}^{n-1} \sigma_{2 k-2 s-1}(m) \sigma_{2 s+1}(n-m) \\
& -2\left[\sum_{s=0}^{k-1}\left(\begin{array}{c}
2 k \\
2 s+1
\end{array}\right) \sum_{m=1}^{n-1} 2^{2 s+1} \sigma_{2 k-2 s-1}(m) \sigma_{2 s+1}\left(\frac{n-m}{2}\right)\right. \\
& \left.+\sum_{s=0}^{k-1}\left(\begin{array}{c}
2 k \\
2 s+1
\end{array}\right) \sum_{m=1}^{n-1} 2^{2 k-2 s-1} \sigma_{2 k-2 s-1}\left(\frac{m}{2}\right) \sigma_{2 s+1}(n-m)\right] \\
& +2^{2 k+2} \sum_{s=0}^{k-1}\left(\begin{array}{c}
2 k \\
2 s+1
\end{array}\right) \sum_{m=1}^{n-1} \sigma_{2 k-2 s-1}\left(\frac{m}{2}\right) \sigma_{2 s+1}\left(\frac{n-m}{2}\right) .
\end{aligned}
$$


From Proposition 1, Lemma 12 and Lemma 16 we obtain that

$$
\begin{aligned}
& \sum_{s=0}^{k-1}\left(\begin{array}{c}
2 k \\
2 s+1
\end{array}\right) \sum_{m=1}^{n-1} \widetilde{\sigma}_{2 k-2 s-1}(m) \widetilde{\sigma}_{2 s+1}(n-m) \\
= & -\frac{1}{2} \sigma_{2 k+1}(n)+\frac{1}{2} \sigma_{2 k}(n)+n \sigma_{2 k-1}(n)-\frac{1}{2 k+1} \sum_{d \mid n} B_{2 k+1}(d+1) \\
& +2^{2 k+1}\left[\frac{1}{2} \sigma_{2 k+1}\left(\frac{n}{2}\right)-\frac{1}{2} \sigma_{2 k}\left(\frac{n}{2}\right)-\frac{n}{2} \sigma_{2 k-1}\left(\frac{n}{2}\right)+\sum_{d \mid \frac{n}{2}} \frac{B_{2 k+1}(d+1)}{2 k+1}\right] \\
& +2^{2 k} \sigma_{2 k+1}\left(\frac{n}{2}\right)+\frac{2^{2 k+1}}{2 k+1} \sum_{d \mid n} B_{2 k+1}\left(\frac{d+1}{2}\right) \\
= & -\frac{1}{2} \sigma_{2 k+1}(n)+\sigma_{2 k+1,0}(n ; 2)+\frac{1}{2} \sigma_{2 k}(n)-\sigma_{2 k, 0}(n ; 2)+n \sigma_{2 k-1}(n) \\
& -2 n \sigma_{2 k-1,0}(n ; 2)+T,
\end{aligned}
$$

where

$$
\begin{aligned}
T:= & -\frac{1}{2 k+1} \sum_{\substack{d \mid n\\
}} B_{2 k+1}(d+1) \\
& +\frac{2^{2 k+1}}{2 k+1}\left(\sum_{\substack{d \mid n \\
d \text { odd }}} B_{2 k+1}\left(\frac{d+1}{2}\right)+\sum_{d \mid \frac{n}{2}} B_{2 k+1}(d+1)\right) \\
= & -\frac{1}{2 k+1} \sum_{\substack{d \mid n \\
d \text { odd }}} B_{2 k+1}(d+1)+\frac{2^{2 k+1}}{2 k+1} \sum_{\substack{d \mid n \\
d \text { odd }}} B_{2 k+1}\left(\frac{d+1}{2}\right) \\
& -\frac{1}{2 k+1} \sum_{\substack{d \mid n \\
d \text { even }}} B_{2 k+1}(d+1)+\frac{2^{2 k+1}}{2 k+1} \sum_{d \mid \frac{n}{2}} B_{2 k+1}(d+1) \\
= & -\frac{1}{2 k+1} \sum_{\substack{d \mid n \\
d \text { odd }}}\left(B_{2 k+1}(d+1)-2^{2 k+1} B_{2 k+1}\left(\frac{d+1}{2}\right)\right) \\
& -\frac{1}{2 k+1} \sum_{\substack{d \mid \frac{n}{2} \\
(}}\left(B_{2 k+1}(2 d+1)-2^{2 k+1} B_{2 k+1}(d+1)\right) .
\end{aligned}
$$

It is well known that

$$
E_{n}(x)=(-1)^{n} E_{n}(1-x)
$$

and

$$
E_{n}(x)=\frac{2}{n+1}\left\{B_{n+1}(x)-2^{n+1} B_{n+1}\left(\frac{x}{2}\right)\right\} .
$$


By (2.9), (2.10) and (2.11), we obtain

$$
\begin{aligned}
T & =\frac{1}{2}\left(\sum_{d \mid \frac{n}{2}} E_{2 k}(-2 d)-\sum_{\substack{d \mid n \\
d \text { odd }}} E_{2 k}(-d)\right) \\
& =\frac{1}{2}\left(\sum_{d \mid \frac{n}{2}} E_{2 k}(2 d+1)-\sum_{\substack{d \mid n \\
d \text { odd }}} E_{2 k}(d+1)\right) \\
& =-\frac{1}{2} \sum_{d \mid n}(-1)^{d-1} E_{2 k}(d+1) .
\end{aligned}
$$

By the definition of $\widetilde{\sigma}_{k}$, we easily seen that

(2.13) $\quad \tilde{\sigma}_{k}(n)=\sigma_{k, 1}(n ; 2)-\sigma_{k, 0}(n ; 2)$ and $\sigma_{k}(n)=\sigma_{k, 1}(n ; 2)+\sigma_{k, 0}(n ; 2)$.

From (2.8), (2.12) and (2.13) we obtain (1.15).

Remark 17. Proposition 6 and Lemma 12 give us two curious formulas replace $\sigma_{s}(n)$ (resp., $\frac{B_{2 k+1}(n)}{2 k+1}$, Faulhaber sum) by $-\widetilde{\sigma}_{s}(n)$ (resp., $-\frac{E_{2 k}(n)}{2}$, alternating sum). See the table below.

Table 1. Convolution sums of divisor functions

\begin{tabular}{|c|c|}
\hline$\sum_{s=1}^{k-1}\left(\begin{array}{c}2 k \\
2 s+1\end{array}\right) \sum_{m=1}^{n-1} \sigma_{2 k-2 s-1}(m) \sigma_{2 s+1}(n-m)$ & $=\frac{1}{2} \sigma_{2 k+1}(n)-\frac{1}{2} \sigma_{2 k}(n)-n \sigma_{2 k-1}(n)$ \\
& $+\frac{1}{2 k+1} \sum_{d \mid n} B_{2 k+1}(d+1)$ \\
\hline$\sum_{s=1}^{k-1}\left(\begin{array}{c}2 k \\
2 s+1\end{array}\right) \sum_{m=1}^{n-1} \widetilde{\sigma}_{2 k-2 s-1}(m) \widetilde{\sigma}_{2 s+1}(n-m)$ & $=-\frac{1}{2} \widetilde{\sigma}_{2 k+1}(n)+\frac{1}{2} \widetilde{\sigma}_{2 k}(n)+n \widetilde{\sigma}_{2 k-1}(n)$ \\
& $-\frac{1}{2} \sum_{d \mid n}(-1)^{d-1} E_{2 k}(d+1)$ \\
\hline
\end{tabular}

Corollary 18. Let $n \geq 2$. Then

$$
\begin{aligned}
& \sum_{s=0}^{k-1}\left(\begin{array}{c}
2 k \\
2 s+1
\end{array}\right) \sum_{m=1}^{n-1} \widetilde{\sigma}_{2 k-2 s-1}(2 m) \widetilde{\sigma}_{2 s+1}(2 n-2 m) \\
= & \frac{1}{2}\left[4 n \widetilde{\sigma}_{2 k-1}(2 n)+\widetilde{\sigma}_{2 k}(2 n)-\widetilde{\sigma}_{2 k+1}(2 n)\right]-\frac{1}{4} \sigma_{2 k+1}^{*}(2 n) \\
& -\frac{1}{2} \sum_{d \mid 2 n}(-1)^{d-1} E_{2 k}(d+1) .
\end{aligned}
$$

Proof. Consider the combinatoric convolution sum

$$
\begin{aligned}
& \sum_{s=0}^{k-1}\left(\begin{array}{c}
2 k \\
2 s+1
\end{array}\right) \sum_{m=1}^{n-1} \widetilde{\sigma}_{2 k-2 s-1}(2 m) \widetilde{\sigma}_{2 s+1}(2 n-2 m) \\
= & \sum_{s=0}^{k-1}\left(\begin{array}{c}
2 k \\
2 s+1
\end{array}\right) \sum_{m=1}^{2 n-1} \widetilde{\sigma}_{2 k-2 s-1}(m) \widetilde{\sigma}_{2 s+1}(2 n-m) \\
& -\sum_{s=0}^{k-1}\left(\begin{array}{c}
2 k \\
2 s+1
\end{array}\right) \sum_{m=1}^{n} \sigma_{2 k-2 s-1}(2 m-1) \sigma_{2 s+1}(2 n-2 m+1) .
\end{aligned}
$$


From [10, (15)] and Proposition 6, we obtain (2.14).

Lemma 19. Let $n \geq 3, m \geq 2$ and $x, y \in \mathbb{R}$. Then

(a)

$$
\begin{aligned}
& \sum_{k=1}^{\left[\frac{n-1}{2}\right]}\left(\begin{array}{c}
n \\
2 k+1
\end{array}\right) B_{2 k+1}(x) y^{n-(2 k+1)} \\
= & \frac{1}{2}\left(B_{n}(x+y)-(-1)^{n} B_{n}(x-y)-n(2 x-1) y^{n-1}\right) .
\end{aligned}
$$

(b)

$$
\sum_{k=1}^{\left[\frac{m}{2}\right]}\left(\begin{array}{c}
m \\
2 k
\end{array}\right) E_{2 k}(x) y^{m-2 k}=\frac{1}{2}\left(E_{m}(x+y)+(-1)^{m} E_{m}(x-y)\right)-y^{m} .
$$

Proof. This lemma follows from (1.2), (2.5) and (2.10).

Proof of Theorem 8. (a) We note that

$$
\sigma_{k, 0}(2 n ; 2)=2^{k} \sigma_{k}(n)
$$

and

$$
\left(\begin{array}{c}
l \\
a, b, c
\end{array}\right)=\left(\begin{array}{l}
l \\
c
\end{array}\right)\left(\begin{array}{c}
a+b \\
a
\end{array}\right)
$$

By Proposition 1, (2.15) and (2.16), we obtain

$$
\begin{aligned}
& Y_{2 l+1}(n, 2 q)=\sum_{c=0}^{l-1}\left(\begin{array}{l}
2 l+1 \\
2 c+1
\end{array}\right)\left\{\sum_{s=0}^{l-c-1}\left(\begin{array}{c}
2 l-2 c \\
2 s+1
\end{array}\right) \sum_{m=1}^{n-1} \sigma_{2 l-2 c-2 s-1,1}(m ; 2) \sigma_{2 s+1,1}(n-m ; 2)\right\} \\
& \times \sigma_{2 c+1,0}(2 q ; 2) \\
&= \sum_{c=0}^{l-1}\left(\begin{array}{l}
2 l+1 \\
2 c+1
\end{array}\right)\left[\frac{1}{4} \sigma_{2 l-2 c+1,0}(n ; 2)+\frac{2^{2 l+1-2 c-1}}{2 l-2 c+1}\left(\sum_{\substack{\alpha \mid n \\
\alpha \text { odd }}} B_{2 l-2 c+1}\left(\frac{\alpha+1}{2}\right)\right)\right] \\
& \quad \times 2^{2 c+1} \sigma_{2 c+1}(q) .
\end{aligned}
$$

Consider the second term of $Y_{2 l+1}(n, 2 q)$, we obtain

$$
\begin{aligned}
& Y_{2 l+1}^{(2)}(n, 2 q) \\
:= & 2^{2 l+1} \sum_{c=0}^{l-1}\left[\frac{(2 l+1) !}{(2 c+1) !(2 l-2 c) !} \cdot \frac{1}{2 l-2 c+1} \sum_{\substack{\alpha \mid n \\
\alpha \text { odd }}} B_{2 l-2 c+1}\left(\frac{\alpha+1}{2}\right)\right] \sigma_{2 c+1}(q) \\
= & \frac{2^{2 l+1}}{2 l+2} \sum_{\substack{\alpha \mid n \\
\alpha \text { odd }}}\left[\sum_{c=0}^{l-1}\left(\begin{array}{l}
2 l+2 \\
2 c+1
\end{array}\right) B_{2 l-2 c+1}\left(\frac{\alpha+1}{2}\right) \sigma_{2 c+1}(q)\right]
\end{aligned}
$$




$$
\begin{aligned}
& =\frac{2^{2 l+1}}{2 l+2} \sum_{\substack{\alpha \mid n \\
\alpha \text { odd }}}\left[\sum_{d \mid q}\left(\sum_{c=0}^{l-1}\left(\begin{array}{l}
2 l+2 \\
2 c+1
\end{array}\right) B_{2 l-2 c+1}\left(\frac{\alpha+1}{2}\right) d^{2 c+1}\right)\right] \\
& =\frac{2^{2 l+1}}{2 l+2} \sum_{\substack{\alpha \mid n \\
\alpha \text { odd }}}\left[\sum_{d \mid q}\left(\sum_{c=1}^{l}\left(\begin{array}{l}
2 l+2 \\
2 c+1
\end{array}\right) B_{2 c+1}\left(\frac{\alpha+1}{2}\right) d^{2 l-2 c+1}\right)\right] .
\end{aligned}
$$

By Lemma 19, we get

$$
\begin{aligned}
& Y_{2 l+1}^{(2)}(n, 2 q) \\
&=\frac{2^{2 l+1}}{2 l+2} \sum_{\substack{\alpha \mid n \\
\alpha \text { odd }}}\left[\sum _ { d | q } \left\{\frac{1}{2}\left(B_{2 l+2}\left(\frac{\alpha+1}{2}+d\right)-B_{2 l+2}\left(\frac{\alpha+1}{2}-d\right)\right)\right.\right. \\
&\left.\left.-(2 l+2)\left(\frac{\alpha+1}{2}-\frac{1}{2}\right) d^{2 l+1}\right\}\right] \\
&= \frac{2^{2 l}}{2 l+2}\left[\sum_{\substack{d|q, \alpha| n \\
\alpha \text { odd }}}\left(B_{2 l+2}\left(\frac{\alpha+1}{2}+d\right)-B_{2 l+2}\left(\frac{\alpha+1}{2}-d\right)\right)\right] \\
&-2^{2 l} \cdot \sigma_{1,1}(n ; 2) \sigma_{2 l+1}(q) .
\end{aligned}
$$

From the binomial theorem we have

$$
\sum_{c=0}^{l-1}\left(\begin{array}{l}
2 l+1 \\
2 c+1
\end{array}\right) x^{2 l-2 c+1} y^{2 c+1}=\frac{x}{2}\left\{(x+y)^{2 l+1}-(x-y)^{2 l+1}\right\}-x y^{2 l+1}
$$

and then we obtain

$$
Y_{2 l+1}^{(1)}(n, 2 q):=2^{2 l-1} \sum_{\alpha\left|\frac{n}{2}, d\right| q} \alpha\left\{(\alpha+d)^{2 l+1}-(\alpha-d)^{2 l+1}\right\}-2^{2 l} \sum_{\alpha\left|\frac{n}{2}, d\right| q} \alpha d^{2 l+1} .
$$

By the property of Bernoulli polynomial,

$$
B_{n}(x+1)-B_{n}(x)=n x^{n-1},
$$

we have

$$
\begin{aligned}
Y_{2 l+1}^{(1)}(n, 2 q):= & \frac{2^{2 l-1}}{2 l+2} \sum_{\alpha\left|\frac{n}{2}, d\right| q} \alpha\left\{B_{2 l+2}(\alpha+d+1)-B_{2 l+2}(\alpha+d)\right. \\
& \left.\quad-B_{2 l+2}(\alpha-d+1)+B_{2 l+2}(\alpha-d)\right\} \\
& -\frac{1}{2} \sigma_{1}\left(\frac{n}{2}\right) \sigma_{2 l+1,0}(2 q ; 2) .
\end{aligned}
$$

Thus, we get

$$
\begin{array}{r}
Y_{2 l+1}(n, 2 q)=\frac{2^{2 l-1}}{2 l+2} \sum_{\alpha\left|\frac{n}{2}, d\right| q} \alpha\left\{B_{2 l+2}(\alpha+d+1)-B_{2 l+2}(\alpha+d)\right. \\
\left.-B_{2 l+2}(\alpha-d+1)+B_{2 l+2}(\alpha-d)\right\}
\end{array}
$$




$$
\begin{aligned}
& +\frac{2^{2 l}}{2 l+2}\left[\sum_{\substack{d|q, \alpha| n \\
\alpha \text { odd }}}\left(B_{2 l+2}\left(\frac{\alpha+1}{2}+d\right)-B_{2 l+2}\left(\frac{\alpha+1}{2}-d\right)\right)\right] \\
& -\frac{1}{2}\left(\sigma_{1,1}(n ; 2)+\sigma_{1}\left(\frac{n}{2}\right)\right) \sigma_{2 l+1,0}(2 q ; 2) .
\end{aligned}
$$

Similarly, by Proposition 1, (2.15) and (2.16), we obtain

$$
\begin{aligned}
Y_{2 l}(n, 2 q):= & \frac{2^{2 l-2}}{2 l+1} \sum_{\alpha\left|\frac{n}{2}, d\right| q} \alpha\left\{B_{2 l+1}(\alpha+d+1)-B_{2 l+1}(\alpha+d)\right. \\
& \left.+B_{2 l+1}(\alpha-d+1)-B_{2 l+1}(\alpha-d)\right\} \\
& +\frac{2^{2 l-1}}{2 l+1}\left[\sum_{\substack{\alpha|q, \alpha| n \\
\alpha \text { odd }}}\left(B_{2 l+1}\left(\frac{\alpha+1}{2}+d\right)+B_{2 l+1}\left(\frac{\alpha+1}{2}-d\right)\right)\right] \\
& -\frac{1}{2}\left(\sigma_{1,1}(n ; 2)+\sigma_{1}\left(\frac{n}{2}\right)\right) \sigma_{2 l, 0}(2 q ; 2) .
\end{aligned}
$$

(b) It is easily checked that $\sigma_{s}^{*}(2 N)=2^{s} \sigma_{s}^{*}(N)$. Using the same method in (a), this completes (b).

(c) First, we consider when $l$ is odd. By Proposition 6 and (2.16), we obtain

$$
\begin{aligned}
U_{2 l+1}(p, q):= & \sum_{c=0}^{l-1}\left(\begin{array}{c}
2 l+1 \\
2 c+1
\end{array}\right)\left\{\sum_{s=0}^{l-c-1}\left(\begin{array}{c}
2 l-2 c \\
2 s+1
\end{array}\right) \sum_{m=1}^{p-1} \widetilde{\sigma}_{2 l-2 c-2 s-1}(m) \widetilde{\sigma}_{2 s+1}(p-m)\right\} \\
& \times \widetilde{\sigma}_{2 c+1}(q) \\
= & \sum_{c=0}^{l-1}\left(\begin{array}{c}
2 l+1 \\
2 c+1
\end{array}\right)\left(p \widetilde{\sigma}_{2 l-2 c-1}(p)+\frac{1}{2} \widetilde{\sigma}_{2 l-2 c}(p)-\frac{1}{2} \widetilde{\sigma}_{2 l-2 c+1}(p)\right. \\
& \left.-\frac{1}{2} \sum_{\alpha \mid p}(-1)^{\alpha-1} E_{2 l-2 c}(\alpha+1)\right) \widetilde{\sigma}_{2 c+1}(q) .
\end{aligned}
$$

It is well known that

$$
\sum_{c=0}^{l-1}\left(\begin{array}{l}
2 l+1 \\
2 c+1
\end{array}\right) x^{2 c+1} y^{2 l-2 c}=\frac{1}{2}\left((x+y)^{2 l+1}+(x-y)^{2 l+1}\right)-x^{2 l+1}
$$

and

$$
2 x^{n}=E_{n}(x+1)+E_{n}(x) .
$$

From Lemma 19, (2.10), (2.19) and (2.20) we deduce that

$$
\begin{aligned}
& \sum_{c=0}^{l-1}\left(\begin{array}{c}
2 l+1 \\
2 c+1
\end{array}\right) p \widetilde{\sigma}_{2 l-2 c-1}(p) \widetilde{\sigma}_{2 c+1}(q) \\
= & \frac{p}{4} \sum_{\alpha|p, d| q}(-1)^{\alpha+d} \frac{1}{\alpha}\left\{E_{2 l+1}(d+\alpha+1)+E_{2 l+1}(d+\alpha)+E_{2 l+1}(d-\alpha+1)\right.
\end{aligned}
$$




$$
\left.+E_{2 l+1}(d-\alpha)\right\}-p \widetilde{\sigma}_{-1}(p) \widetilde{\sigma}_{2 l+1}(q),
$$

$$
\begin{aligned}
& \frac{1}{2} \sum_{c=0}^{l-1}\left(\begin{array}{c}
2 l+1 \\
2 c+1
\end{array}\right) \widetilde{\sigma}_{2 l-2 c}(p) \widetilde{\sigma}_{2 c+1}(q) \\
= & \frac{1}{8} \sum_{\alpha|p, d| q}(-1)^{\alpha+d}\left\{E_{2 l+1}(d+\alpha+1)+E_{2 l+1}(d+\alpha)+E_{2 l+1}(d-\alpha+1)\right. \\
& \left.+E_{2 l+1}(d-\alpha)\right\}-\frac{1}{2} \widetilde{\sigma}_{2 l+1}(q) \widetilde{\sigma}_{0}(p), \\
- & \frac{1}{2} \sum_{c=0}^{l-1}\left(\begin{array}{l}
2 l+1 \\
2 c+1
\end{array}\right) \widetilde{\sigma}_{2 l-2 c+1}(p) \widetilde{\sigma}_{2 c+1}(q) \\
= & \frac{1}{8} \sum_{\alpha|p, d| q}(-1)^{\alpha+d} \alpha\left\{E_{2 l+1}(d+\alpha+1)+E_{2 l+1}(d+\alpha)+E_{2 l+1}(d-\alpha+1)\right. \\
& \left.+E_{2 l+1}(d-\alpha)\right\}+\frac{1}{2} \widetilde{\sigma}_{1}(p) \widetilde{\sigma}_{2 l+1}(q), \\
& -\frac{1}{2} \sum_{c=0}^{l-1}\left(\begin{array}{l}
2 l+1 \\
2 c+1
\end{array}\right)\left(\sum_{\alpha \mid p}(-1)^{\alpha-1} E_{2 l-2 c}(\alpha+1)\right) \widetilde{\sigma}_{2 c+1}(q) \\
= & -\frac{1}{4} \sum_{\alpha|p, d| q}(-1)^{\alpha+d}\left\{E_{2 l+1}(d+\alpha+1)+E_{2 l+1}(d-\alpha)\right\}+\frac{1}{2} \widetilde{\sigma}_{0}(p) \widetilde{\sigma}_{2 l+1}(q) .
\end{aligned}
$$

By a routine calculation, we get

$$
\begin{aligned}
U_{2 l+1}(p, q)= & \frac{1}{8} \sum_{\substack{\alpha|p \\
d| q}} \frac{(-1)^{\alpha+d}}{\alpha}\left[\left(2 p+\alpha-\alpha^{2}\right)\left\{E_{2 l+1}(d+\alpha)+E_{2 l+1}(d-\alpha+1)\right\}\right. \\
& \left.+\left(2 p-\alpha-\alpha^{2}\right)\left\{E_{2 l+1}(d+\alpha+1)+E_{2 l+1}(d-\alpha)\right\}\right] \\
& +\frac{1}{2} \widetilde{\sigma}_{2 l+1}(q)\left\{\widetilde{\sigma}_{1}(p)-2 p \widetilde{\sigma}_{-1}(p)\right\} .
\end{aligned}
$$

Similarly, when $l$ is even, we have then the result.

Remark 20. In [14, Theorem 3] we recall that

$$
\begin{aligned}
& \sum_{\substack{a+b+c=2 l+1 \\
a, b, c \text { odd }}} a\left(\begin{array}{c}
2 l+1 \\
a, b, c
\end{array}\right) \sum_{\substack{m_{1}+m_{2}+m_{3}=N \\
m_{3} \text { even }}}(-1)^{m_{1}+1} \sigma_{a}^{*}\left(m_{1}\right) \sigma_{b}^{*}\left(m_{2}\right) \sigma_{c}^{*}\left(m_{3}\right) \\
= & \frac{(2 l+1) N}{32}\left\{\sigma_{2 l+1}^{*}(N)-2 N \sigma_{2 l-1}^{*}(N)\right\}
\end{aligned}
$$

with $N \geq 4$ and $l \in \mathbb{N}$. Theorem 8 are analogous results of this identity. 


\section{Proof of second main results: Theorem 9 and Theorem 11}

Before proving Theorem 9 we note some easy lemmas for convolution Bernoulli polynomials.

Lemma 21. Let $m \geq 2$. Then we have

(a)

$$
\begin{aligned}
& \sum_{k=1}^{m-1}\left(\begin{array}{c}
2 m \\
2 k
\end{array}\right) \frac{B_{2 k+1}(x)}{2 k+1} \frac{B_{2 m-2 k+1}(y)}{2 m-2 k+1} \\
= & \frac{1}{4 m+2}\left\{(x+y-1) B_{2 m+1}(x+y)+(x-y) B_{2 m+1}(y-x)\right. \\
& \left.-(2 x-1) B_{2 m+1}(y)-(2 y-1) B_{2 m+1}(x)\right\} \\
& -\frac{1}{4 m+4}\left\{B_{2 m+2}(x+y)-B_{2 m+2}(y-x)\right\} .
\end{aligned}
$$

(b)

$$
\begin{aligned}
& \sum_{k=1}^{m-1}\left(\begin{array}{c}
2 m \\
2 k
\end{array}\right) E_{2 k}(x) E_{2 m-2 k}(y) \\
= & (1-x-y) E_{2 m}(x+y)+(x-y) E_{2 m}(1-x+y) \\
& -E_{2 m}(x)-E_{2 m}(y)+E_{2 m+1}(x+y)+E_{2 m+1}(1-x+y) .
\end{aligned}
$$

Proof. (a) By [5, p. 158], we see that

$$
\begin{aligned}
& \sum_{k=0}^{m}\left(\begin{array}{c}
m \\
k
\end{array}\right) \frac{B_{k+1}(x)}{k+1} \frac{B_{m-k+1}(y)}{m-k+1} \\
= & (x+y-1) \frac{B_{m+1}(x+y)}{m+1}-\frac{B_{m+2}(x+y)}{m+2} \\
& -\frac{B_{m+2}(x)}{(m+1)(m+2)}-\frac{B_{m+2}(y)}{(m+1)(m+2)}, \\
& \sum_{k=0}^{m}(-1)^{k}\left(\begin{array}{c}
m \\
k
\end{array}\right) \frac{B_{k+1}(x)}{k+1} \frac{B_{m-k+1}(y)}{m-k+1} \\
= & (x-y) \frac{B_{m+1}(y-x)}{m+1}+\frac{B_{m+2}(y-x)}{m+2} \\
& +\frac{B_{m+2}(1-x)}{(m+1)(m+2)}+\frac{B_{m+2}(y)}{(m+1)(m+2)}
\end{aligned}
$$

with $m$ a positive integer. If we set $m=2 m$ in (3.1) and (3.2), summing for (3.1) and (3.2), the result follows. Using [5, p. 150] or [20, (20)], we see that

$$
\sum_{k=0}^{m}\left(\begin{array}{c}
m \\
k
\end{array}\right) E_{k}(x) E_{m-k}(y)=2(1-x-y) E_{2 m}(x+y)+2 E_{2 m+1}(x+y) \text {. }
$$


Set $x=1-x$ in $(3.3)$, and using $(2.10)$, we get

$\sum_{k=0}^{m}\left(\begin{array}{c}m \\ k\end{array}\right)(-1)^{k} E_{k}(x) E_{m-k}(y)=2(x-y) E_{2 m}(1-x+y)+2 E_{2 m+1}(1-x+y)$.

In a similar way, we get (b).

Proof of Theorem 9. It is well known that

$$
\begin{aligned}
& \left(\begin{array}{c}
2 m \\
2 k
\end{array}\right)\left(\begin{array}{c}
2 k \\
2 s+1
\end{array}\right)\left(\begin{array}{c}
2 m-2 k \\
2 s^{\prime}+1
\end{array}\right) \\
= & \frac{(2 m) !}{(2 s+1) !(2 k-2 s-1) !\left(2 s^{\prime}+1\right) !\left(2 m-2 k-2 s^{\prime}-1\right) !} \\
= & \left(\begin{array}{c}
2 m \\
2 s+1,2 k-2 s-1,2 s^{\prime}+1,2 m-2 k-2 s^{\prime}-1
\end{array}\right) .
\end{aligned}
$$

Now if we set $a=2 s+1, b=2 k-2 s-1, c=2 s^{\prime}+1, d=2 m-2 k-2 s^{\prime}-1$ and (1.16) becomes

$(3.5)$

$$
\begin{gathered}
\sum_{\substack{1 \leq l \leq p-1,1 \leq l^{\prime} \leq q-1 \\
a, b, c, d \text { odd }, a+b+c+d=2 m}}\left(\begin{array}{c}
2 m \\
a, b, c, d
\end{array}\right) \sigma_{a, 1}(l ; 2) \sigma_{b, 1}(p-l ; 2) \sigma_{c, 1}\left(l^{\prime} ; 2\right) \sigma_{d, 1}\left(q-l^{\prime} ; 2\right) \\
=\sum_{k=1}^{m-1}\left(\begin{array}{c}
2 m \\
2 k
\end{array}\right)\left\{\sum_{s=0}^{k-1}\left(\begin{array}{c}
2 k \\
2 s+1
\end{array}\right) \sum_{l=1}^{p-1} \sigma_{2 k-2 s-1,1}(l ; 2) \sigma_{2 s+1,1}(p-l ; 2)\right\} \\
\quad \times\left\{\sum_{s^{\prime}=0}^{m-k-1}\left(\begin{array}{c}
2 m-2 k \\
2 s^{\prime}+1
\end{array}\right) \sum_{l^{\prime}=1}^{q-1} \sigma_{2 m-2 k-2 s^{\prime}-1,1}\left(l^{\prime} ; 2\right) \sigma_{2 s^{\prime}+1,1}\left(q-l^{\prime} ; 2\right)\right\} .
\end{gathered}
$$

Thus, from Proposition 1, we consider 4 terms in (3.5) below:

$$
\begin{aligned}
& C_{1}:=\sum_{\substack{d\left|p, d^{\prime}\right| q \\
d, d^{\prime} \text { odd }}}^{m-1}\left(\begin{array}{c}
2 m \\
2 k
\end{array}\right) 2^{2 m} \frac{B_{2 k+1}\left(\frac{d+1}{2}\right)}{2 k+1} \cdot \frac{B_{2 m-2 k+1}\left(\frac{d^{\prime}+1}{2}\right)}{2 m-2 k+1}, \\
& C_{2}:=\frac{1}{16} \sum_{k=1}^{m-1}\left(\begin{array}{c}
2 m \\
2 k
\end{array}\right) \sigma_{2 k+1,0}(p ; 2) \sigma_{2 m-2 k+1,0}(q ; 2), \\
& C_{3}:=\frac{1}{4} \sum_{k=1}^{m-1}\left(\begin{array}{c}
2 m \\
2 k
\end{array}\right) 2^{2 m-2 k} \sigma_{2 k+1,0}(p ; 2) \sum_{\substack{d^{\prime} \mid q \\
d^{\prime} \text { odd }}} \frac{B_{2 m-2 k+1}\left(\frac{d^{\prime}+1}{2}\right)}{2 m-2 k+1}, \\
& C_{4}:=\frac{1}{4} \sum_{k=1}^{m-1}\left(\begin{array}{c}
2 m \\
2 k
\end{array}\right) 2^{2 k} \sigma_{2 m-2 k+1,0}(q ; 2) \sum_{\substack{d \mid p \\
d \text { odd }}} \frac{B_{2 k+1}\left(\frac{d+1}{2}\right)}{2 k+1} .
\end{aligned}
$$


Now, first, we consider $C_{1}$. Set $x=(d+1) / 2$ and $y=\left(d^{\prime}+1\right) / 2$ in Lemma 21. Then we have

$$
\begin{gathered}
C_{1}=\frac{2^{2 m-2}}{2 m+1} \sum_{\begin{array}{c}
d\left|p, d^{\prime}\right| q \\
d, d^{\prime} \text { odd }
\end{array}}\left\{\left(d+d^{\prime}\right) B_{2 m+1}\left(\frac{d+d^{\prime}}{2}+1\right)+\left(d-d^{\prime}\right) B_{2 m+1}\left(\frac{d^{\prime}-d}{2}\right)\right. \\
\left.-2 d B_{2 m+1}\left(\frac{d^{\prime}+1}{2}\right)-2 d^{\prime} B_{2 m+1}\left(\frac{d+1}{2}\right)\right\} \\
-\frac{2^{2 m-2}}{m+1} \sum_{\begin{array}{c}
d\left|p, d^{\prime}\right| q \\
d, d^{\prime} \text { odd }
\end{array}}\left\{B_{2 m+2}\left(\frac{d+d^{\prime}}{2}+1\right)-B_{2 m+2}\left(\frac{d^{\prime}-d}{2}\right)\right\} .
\end{gathered}
$$

Second, we consider $C_{2}$. Using the binomial theorem, the equation (2.15) and the difference formula of Bernoulli polynomials, then we obtain

$$
\sum_{k=1}^{m-1}\left(\begin{array}{c}
2 m \\
2 k
\end{array}\right) d^{2 k+1} d^{2 m-2 k+1}=\frac{d d^{\prime}}{2}\left\{\left(d+d^{\prime}\right)^{2 m}+\left(d-d^{\prime}\right)^{2 m}\right\}-d d^{2 m+1}-d^{2 m+1} d^{\prime},
$$

$$
\begin{aligned}
C_{2}= & 2^{2 m-2} \sum_{d\left|\frac{p}{2}, d^{\prime}\right| \frac{q}{2}} \sum_{k=1}^{m-1}\left(\begin{array}{c}
2 m \\
2 k
\end{array}\right) d^{2 k+1} d^{\prime 2 m-2 k+1} \\
= & 2^{2 m-3} \sum_{d\left|\frac{p}{2}, d^{\prime}\right| \frac{q}{2}} d d^{\prime}\left\{\left(d+d^{\prime}\right)^{2 m}+\left(d-d^{\prime}\right)^{2 m}-2 d^{\prime 2 m}-2 d^{2 m}\right\} \\
= & \frac{2^{2 m-3}}{2 m+1} \sum_{\substack{d\left|\frac{p}{2} \\
d^{\prime}\right| \frac{q}{2}}} d d^{\prime}\left\{B_{2 m+1}\left(d+d^{\prime}+1\right)-B_{2 m+1}\left(d+d^{\prime}\right)+B_{2 m+1}\left(d-d^{\prime}+1\right)\right. \\
& \left.-B_{2 m+1}\left(d-d^{\prime}\right)\right\}-\frac{1}{16}\left\{\sigma_{2 m+1,0}(p ; 2) \sigma_{1,0}(q ; 2)+\sigma_{1,0}(p ; 2) \sigma_{2 m+1,0}(q ; 2)\right\} .
\end{aligned}
$$

To find a formula of $C_{3}$, we need an addition formula of Bernoulli polynomials in (1.2).

$$
\begin{aligned}
C_{3} & =\frac{2^{2 m-1}}{2 m+1} \sum_{\substack{d\left|\frac{p}{2}, d^{\prime}\right| q, d^{\prime} \text { odd }}} \sum_{k=1}^{m-1}\left(\begin{array}{c}
2 m+1 \\
2 k
\end{array}\right) d^{2 k+1} B_{2 m-2 k+1}\left(\frac{d^{\prime}+1}{2}\right) \\
& =\frac{2^{2 m-1}}{2 m+1} \sum_{\substack{d\left|\frac{p}{2}, d^{\prime}\right| q, d^{\prime} \text { odd }}} d\left[\sum_{k=1}^{m-1}\left(\begin{array}{c}
2 m+1 \\
2 k+1
\end{array}\right) B_{2 k+1}\left(\frac{d^{\prime}+1}{2}\right) d^{2 m+1-(2 k+1)}\right] .
\end{aligned}
$$


Using the formula

$$
\begin{aligned}
& \sum_{k=1}^{m-1}\left(\begin{array}{c}
2 m+1 \\
2 k+1
\end{array}\right) B_{2 k+1}(x) y^{2 m+1-(2 k+1)} \\
= & \frac{1}{2}\left(B_{2 m+1}(x+y)+B_{2 m+1}(x-y)-(2 m+1)(2 x-1) y^{2 m}-2 B_{2 m+1}(x)\right)
\end{aligned}
$$

we obtain

$$
\begin{aligned}
C_{3}= & \frac{2^{2 m-2}}{2 m+1} \sum_{\substack{d\left|\frac{p}{2}, d^{\prime}\right| q \\
d^{\prime} \text { odd }}} d\left[B_{2 m+1}\left(\frac{d^{\prime}+1+2 d}{2}\right)+B_{2 m+1}\left(\frac{d^{\prime}+1-2 d}{2}\right)\right] \\
& -\frac{2^{2 m-2}}{2 m+1} \sigma_{1,0}(p ; 2) \sum_{\substack{d^{\prime} \mid q \\
d^{\prime} \text { odd }}} B_{2 m+1}\left(\frac{d^{\prime}+1}{2}\right)-\frac{1}{8} \sigma_{2 m+1,0}(p ; 2) \sigma_{1,1}(q ; 2) .
\end{aligned}
$$

Since $C_{4}$ is a symmetric form of $C_{3}$, we deduce

$$
\begin{aligned}
C_{4}= & \frac{2^{2 m-2}}{2 m+1} \sum_{\substack{d^{\prime}\left|\frac{q}{2}, d\right| p \\
d \text { odd }}} d^{\prime}\left[B_{2 m+1}\left(\frac{d+1+2 d^{\prime}}{2}\right)+B_{2 m+1}\left(\frac{d+1-2 d^{\prime}}{2}\right)\right] \\
& -\frac{2^{2 m-2}}{2 m+1} \sigma_{1,0}(q ; 2) \sum_{\substack{d \mid p \\
d \text { odd }}} B_{2 m+1}\left(\frac{d+1}{2}\right)-\frac{1}{8} \sigma_{2 m+1,0}(q ; 2) \sigma_{1,1}(p ; 2) .
\end{aligned}
$$

Summing $C_{i}(i=1, \ldots, 4)$, we derive the theorem.

Remark 22. For a positive $n \in \mathbb{N}-\{1\}$, let $S_{a, b}^{k}(n):=b^{k}+(a+b)^{k}+(2 a+$ $b)^{k}+\cdots+(a(n-1)+b)^{k}$. We recall $[1,(11)]$ that

$$
S_{a, b}^{k}(n)=\frac{a^{k}}{k+1}\left(\left[B_{k+1}\left(n+\frac{b}{a}\right)-B_{k+1}\right]-\left[B_{k+1}\left(\frac{b}{a}\right)-B_{k+1}\right]\right) .
$$

By (3.10), (1.9) and Example 10, we have

$$
\sum_{s=0}^{k-1}\left(\begin{array}{c}
2 k \\
2 s+1
\end{array}\right) \sum_{m=1}^{p-1} \sigma_{2 k-2 s-1,1}(m ; 2) \sigma_{2 s+1,1}(p-m ; 2)=S_{2,0}^{2 k}\left(\frac{p+1}{2}\right)
$$

and

$$
\begin{aligned}
& \sum_{\substack{1 \leq l, l^{\prime} \leq p-1 \\
a, b, c, d \text { odd }}}\left(\begin{array}{c}
2 m \\
a, b, c, d
\end{array}\right) \sigma_{a, 1}(l ; 2) \sigma_{b, 1}(p-l ; 2) \sigma_{c, 1}\left(l^{\prime} ; 2\right) \sigma_{d, 1}\left(p-l^{\prime} ; 2\right) \\
= & \frac{p}{2} S_{2,0}^{2 m}(p+1)-p S_{2,0}^{2 m}\left(\frac{p+1}{2}\right)-\frac{1}{4} S_{2,0}^{2 m+1}(p+1)
\end{aligned}
$$

with $p$ an odd prime integer. 
Proof of Theorem 11. By (3.4) and Proposition 6, we get

$$
\begin{aligned}
& \sum_{\substack{1 \leq l \leq p-1,1 \leq l^{\prime} \leq q-1 \\
a, b, c, d \text { odd } \\
a+b+c+d=2 m}}\left(\begin{array}{c}
2 m \\
a, b, c, d
\end{array}\right) \widetilde{\sigma}_{a}(l) \widetilde{\sigma}_{b}(p-l) \widetilde{\sigma}_{c}\left(l^{\prime}\right) \widetilde{\sigma}_{d}\left(q-l^{\prime}\right) \\
& =\sum_{k=1}^{m-1}\left(\begin{array}{c}
2 m \\
2 k
\end{array}\right)\left(\sum_{s=0}^{k-1}\left(\begin{array}{c}
2 k \\
2 s+1
\end{array}\right) \sum_{l=1}^{p-1} \widetilde{\sigma}_{2 k-2 s-1}(l) \widetilde{\sigma}_{2 s+1}(p-l)\right) \\
& \times\left(\sum_{s^{\prime}=0}^{m-k-1}\left(\begin{array}{c}
2 m-2 k \\
2 s+1
\end{array}\right) \sum_{l^{\prime}=1}^{q-1} \widetilde{\sigma}_{2 m-2 k-2 s^{\prime}-1}\left(l^{\prime}\right) \widetilde{\sigma}_{2 s^{\prime}+1}\left(q-l^{\prime}\right)\right) \\
& =\sum_{k=1}^{m-1}\left(\begin{array}{c}
2 m \\
2 k
\end{array}\right)\left(-\frac{1}{2} \widetilde{\sigma}_{2 k+1}(p)+\frac{1}{2} \widetilde{\sigma}_{2 k}(p)+p \widetilde{\sigma}_{2 k-1}(p)-\frac{1}{2} \sum_{d \mid p}(-1)^{d-1} E_{2 k}(d+1)\right) \\
& \times\left(-\frac{1}{2} \widetilde{\sigma}_{2 m-2 k+1}(q)+\frac{1}{2} \widetilde{\sigma}_{2 m-2 k}(q)+q \widetilde{\sigma}_{2 m-2 k-1}(q)\right. \\
& \left.-\frac{1}{2} \sum_{d^{\prime} \mid q}(-1)^{d^{\prime}-1} E_{2 m-2 k}\left(d^{\prime}+1\right)\right) \text {. }
\end{aligned}
$$

We consider the sum

$$
D_{1}:=\frac{1}{4} \sum_{k=1}^{m-1}\left(\begin{array}{c}
2 m \\
2 k
\end{array}\right) \sum_{\substack{d\left|p \\
d^{\prime}\right| q}}(-1)^{d-1}(-1)^{d^{\prime}-1} E_{2 k}(d+1) E_{2 m-2 k}\left(d^{\prime}+1\right)
$$

by Lemma 21(b) we have

$$
\begin{aligned}
D_{1}= & \frac{1}{4} \sum_{\substack{d\left|p \\
d^{\prime}\right| q}}(-1)^{d+d^{\prime}}\left\{-\left(d+d^{\prime}+1\right) E_{2 m}\left(d+d^{\prime}+2\right)+\left(d-d^{\prime}\right) E_{2 m}\left(d^{\prime}-d+1\right)\right. \\
& \left.\quad-E_{2 m}(d+1)-E_{2 m}\left(d^{\prime}+1\right)+E_{2 m+1}\left(d+d^{\prime}+2\right)+E_{2 m+1}\left(d^{\prime}-d+1\right)\right\} .
\end{aligned}
$$

Here, we have

$$
\begin{aligned}
\sum_{\substack{d\left|p \\
d^{\prime}\right| q}}(-1)^{d+d^{\prime}} E_{2 m}(d+1) & =\left(\sum_{d^{\prime} \mid q}(-1)^{d^{\prime}-1} \cdot 1\right) \cdot\left(\sum_{d \mid p}(-1)^{d-1} E_{2 m}(d+1)\right) \\
& =\widetilde{\sigma}_{0}(q) \sum_{d \mid p}(-1)^{d-1} E_{2 m}(d+1) .
\end{aligned}
$$

Hence,

$$
D_{1}=\frac{1}{4} \sum_{\substack{d\left|p \\ d^{\prime}\right| q}}(-1)^{d+d^{\prime}}\left\{-\left(d+d^{\prime}+1\right) E_{2 m}\left(d+d^{\prime}+2\right)+\left(d-d^{\prime}\right) E_{2 m}\left(d^{\prime}-d+1\right)\right.
$$




$$
\begin{gathered}
\left.+E_{2 m+1}\left(d+d^{\prime}+2\right)+E_{2 m+1}\left(d^{\prime}-d+1\right)\right\} \\
-\frac{1}{4} \widetilde{\sigma}_{0}(q) \sum_{d \mid p}(-1)^{d-1} E_{2 m}(d+1)-\frac{1}{4} \widetilde{\sigma}_{0}(p) \sum_{d^{\prime} \mid q}(-1)^{d^{\prime}-1} E_{2 m}\left(d^{\prime}+1\right) .
\end{gathered}
$$

By the same method in Theorem 8(c) and Theorem 9, we derive the following 15 terms in (3.11) below:

$$
\begin{aligned}
& D_{2}:=\frac{1}{4} \sum_{k=1}^{m-1}\left(\begin{array}{c}
2 m \\
2 k
\end{array}\right) \widetilde{\sigma}_{2 k+1}(p) \widetilde{\sigma}_{2 m-2 k+1}(q) \\
& =\frac{1}{8} \sum_{\substack{d\left|p \\
d^{\prime}\right| q}}(-1)^{d+d^{\prime}} d d^{\prime}\left\{\left(d+d^{\prime}\right)^{2 m}+\left(d-d^{\prime}\right)^{2 m}\right\} \\
& -\frac{1}{4}\left\{\widetilde{\sigma}_{2 m+1}(p) \widetilde{\sigma}_{1}(q)+\widetilde{\sigma}_{2 m+1}(q) \widetilde{\sigma}_{1}(p)\right\}, \\
& D_{3}:=-\frac{1}{4} \sum_{k=1}^{m-1}\left(\begin{array}{c}
2 m \\
2 k
\end{array}\right) \widetilde{\sigma}_{2 k+1}(p) \widetilde{\sigma}_{2 m-2 k}(q) \\
& =-\frac{1}{8} \sum_{\substack{d\left|p \\
d^{\prime}\right| q}}(-1)^{d+d^{\prime}} d\left\{\left(d+d^{\prime}\right)^{2 m}+\left(d-d^{\prime}\right)^{2 m}\right\} \\
& +\frac{1}{4}\left\{\widetilde{\sigma}_{2 m+1}(p) \widetilde{\sigma}_{0}(q)+\widetilde{\sigma}_{2 m}(q) \widetilde{\sigma}_{1}(p)\right\}, \\
& D_{4}:=-\frac{1}{2} \sum_{k=1}^{m-1}\left(\begin{array}{c}
2 m \\
2 k
\end{array}\right) q \widetilde{\sigma}_{2 k+1}(p) \widetilde{\sigma}_{2 m-2 k-1}(q) \\
& =-\frac{q}{4} \sum_{\substack{d\left|p \\
d^{\prime}\right| q}}(-1)^{d+d^{\prime}} \frac{d}{d^{\prime}}\left\{\left(d+d^{\prime}\right)^{2 m}+\left(d-d^{\prime}\right)^{2 m}\right\} \\
& +\frac{q}{2}\left\{\widetilde{\sigma}_{2 m+1}(p) \widetilde{\sigma}_{-1}(q)+\widetilde{\sigma}_{2 m-1}(q) \widetilde{\sigma}_{1}(p)\right\}, \\
& D_{5}:=-\frac{1}{4} \sum_{k=1}^{m-1}\left(\begin{array}{c}
2 m \\
2 k
\end{array}\right) \widetilde{\sigma}_{2 k}(p) \widetilde{\sigma}_{2 m-2 k+1}(q) \\
& =-\frac{1}{8} \sum_{\substack{d\left|p \\
d^{\prime}\right| q}}(-1)^{d+d^{\prime}} d^{\prime}\left\{\left(d+d^{\prime}\right)^{2 m}+\left(d-d^{\prime}\right)^{2 m}\right\} \\
& +\frac{1}{4}\left\{\widetilde{\sigma}_{2 m+1}(q) \widetilde{\sigma}_{0}(p)+\widetilde{\sigma}_{2 m}(p) \widetilde{\sigma}_{1}(q)\right\}, \\
& D_{6}:=\frac{1}{4} \sum_{k=1}^{m-1}\left(\begin{array}{c}
2 m \\
2 k
\end{array}\right) \widetilde{\sigma}_{2 k}(p) \widetilde{\sigma}_{2 m-2 k}(q)
\end{aligned}
$$




$$
\begin{aligned}
& =\frac{1}{8} \sum_{\substack{d\left|p \\
d^{\prime}\right| q}}(-1)^{d+d^{\prime}}\left\{\left(d+d^{\prime}\right)^{2 m}+\left(d-d^{\prime}\right)^{2 m}\right\} \\
& -\frac{1}{4}\left\{\widetilde{\sigma}_{2 m}(p) \widetilde{\sigma}_{0}(q)+\widetilde{\sigma}_{2 m}(q) \widetilde{\sigma}_{0}(p)\right\}, \\
& D_{7}:=\frac{1}{2} \sum_{k=1}^{m-1}\left(\begin{array}{c}
2 m \\
2 k
\end{array}\right) q \widetilde{\sigma}_{2 k}(p) \widetilde{\sigma}_{2 m-2 k-1}(q) \\
& =\frac{q}{4} \sum_{\substack{d\left|p \\
d^{\prime}\right| q}}(-1)^{d+d^{\prime}} \frac{1}{d^{\prime}}\left\{\left(d+d^{\prime}\right)^{2 m}+\left(d-d^{\prime}\right)^{2 m}\right\} \\
& -\frac{q}{2}\left\{\widetilde{\sigma}_{2 m}(p) \widetilde{\sigma}_{-1}(q)+\widetilde{\sigma}_{2 m-1}(q) \widetilde{\sigma}_{0}(p)\right\}, \\
& D_{8}:=-\frac{1}{2} \sum_{k=1}^{m-1}\left(\begin{array}{c}
2 m \\
2 k
\end{array}\right) p \widetilde{\sigma}_{2 k-1}(p) \widetilde{\sigma}_{2 m-2 k+1}(q) \\
& =-\frac{p}{4} \sum_{\substack{d\left|p \\
d^{\prime}\right| q}}(-1)^{d+d^{\prime}} \frac{d^{\prime}}{d}\left\{\left(d+d^{\prime}\right)^{2 m}+\left(d-d^{\prime}\right)^{2 m}\right\} \\
& +\frac{p}{2}\left\{\widetilde{\sigma}_{2 m+1}(q) \widetilde{\sigma}_{-1}(p)+\widetilde{\sigma}_{2 m-1}(p) \widetilde{\sigma}_{1}(q)\right\}, \\
& D_{9}:=\frac{1}{2} \sum_{k=1}^{m-1}\left(\begin{array}{c}
2 m \\
2 k
\end{array}\right) p \widetilde{\sigma}_{2 k-1}(p) \widetilde{\sigma}_{2 m-2 k}(q) \\
& =\frac{p}{4} \sum_{\substack{d\left|p \\
d^{\prime}\right| q}}(-1)^{d+d^{\prime}} \frac{1}{d}\left\{\left(d+d^{\prime}\right)^{2 m}+\left(d-d^{\prime}\right)^{2 m}\right\} \\
& -\frac{p}{2}\left\{\widetilde{\sigma}_{2 m}(q) \widetilde{\sigma}_{-1}(p)+\widetilde{\sigma}_{2 m-1}(p) \widetilde{\sigma}_{0}(q)\right\}, \\
& D_{10}:=\sum_{k=1}^{m-1}\left(\begin{array}{c}
2 m \\
2 k
\end{array}\right) p \widetilde{\sigma}_{2 k-1}(p) q \widetilde{\sigma}_{2 m-2 k-1}(q) \\
& =\frac{p q}{2} \sum_{\substack{d\left|p \\
d^{\prime}\right| q}}(-1)^{d+d^{\prime}} \frac{1}{d d^{\prime}}\left\{\left(d+d^{\prime}\right)^{2 m}+\left(d-d^{\prime}\right)^{2 m}\right\} \\
& -p q\left\{\widetilde{\sigma}_{2 m-1}(p) \widetilde{\sigma}_{-1}(q)+\widetilde{\sigma}_{2 m-1}(q) \widetilde{\sigma}_{-1}(p)\right\}, \\
& D_{11}:=\frac{1}{4} \sum_{k=1}^{m-1}\left(\begin{array}{c}
2 m \\
2 k
\end{array}\right) \sum_{d^{\prime} \mid q}(-1)^{d^{\prime}-1} \widetilde{\sigma}_{2 k+1}(p) E_{2 m-2 k}\left(d^{\prime}+1\right) \\
& =\frac{1}{8} \sum_{\substack{d\left|p \\
d^{\prime}\right| q}}(-1)^{d+d^{\prime}} d\left\{E_{2 m}\left(d+d^{\prime}+1\right)+E_{2 m}\left(d^{\prime}-d+1\right)\right\}
\end{aligned}
$$




$$
\begin{aligned}
& -\frac{1}{4}\left\{\widetilde{\sigma}_{1}(p) \sum_{d^{\prime} \mid q}(-1)^{d^{\prime}-1} E_{2 m}\left(d^{\prime}+1\right)+\widetilde{\sigma}_{2 m+1}(p) \widetilde{\sigma}_{0}(q)\right\} \\
& D_{12}:=-\frac{1}{4} \sum_{k=1}^{m-1}\left(\begin{array}{c}
2 m \\
2 k
\end{array}\right) \sum_{d^{\prime} \mid q}(-1)^{d^{\prime}-1} \widetilde{\sigma}_{2 k}(p) E_{2 m-2 k}\left(d^{\prime}+1\right) \\
& =-\frac{1}{8} \sum_{\substack{d\left|p \\
d^{\prime}\right| q}}(-1)^{d+d^{\prime}}\left\{E_{2 m}\left(d+d^{\prime}+1\right)+E_{2 m}\left(d^{\prime}-d+1\right)\right\} \\
& +\frac{1}{4}\left\{\widetilde{\sigma}_{0}(p) \sum_{d^{\prime} \mid q}(-1)^{d^{\prime}-1} E_{2 m}\left(d^{\prime}+1\right)+\widetilde{\sigma}_{2 m}(p) \widetilde{\sigma}_{0}(q)\right\}, \\
& D_{13}:=-\frac{1}{2} \sum_{k=1}^{m-1}\left(\begin{array}{c}
2 m \\
2 k
\end{array}\right) \sum_{d^{\prime} \mid q}(-1)^{d^{\prime}-1} p \widetilde{\sigma}_{2 k-1}(p) E_{2 m-2 k}\left(d^{\prime}+1\right) \\
& =-\frac{p}{4} \sum_{\substack{d\left|p \\
d^{\prime}\right| q}}(-1)^{d+d^{\prime}} \frac{1}{d}\left\{E_{2 m}\left(d+d^{\prime}+1\right)+E_{2 m}\left(d^{\prime}-d+1\right)\right\} \\
& +\frac{p}{2}\left\{\widetilde{\sigma}_{-1}(p) \sum_{d^{\prime} \mid q}(-1)^{d^{\prime}-1} E_{2 m}\left(d^{\prime}+1\right)+\widetilde{\sigma}_{2 m-1}(p) \widetilde{\sigma}_{0}(q)\right\}, \\
& D_{14}:=\frac{1}{4} \sum_{k=1}^{m-1}\left(\begin{array}{c}
2 m \\
2 k
\end{array}\right) \sum_{d \mid p}(-1)^{d-1} E_{2 k}(d+1) \widetilde{\sigma}_{2 m-2 k+1}(q) \\
& =\frac{1}{8} \sum_{\substack{d\left|p \\
d^{\prime}\right| q}}(-1)^{d+d^{\prime}} d^{\prime}\left\{E_{2 m}\left(d+d^{\prime}+1\right)+E_{2 m}\left(d-d^{\prime}+1\right)\right\} \\
& -\frac{1}{4}\left\{\widetilde{\sigma}_{1}(q) \sum_{d \mid p}(-1)^{d-1} E_{2 m}(d+1)+\widetilde{\sigma}_{2 m+1}(q) \widetilde{\sigma}_{0}(p)\right\}, \\
& D_{15}:=-\frac{1}{4} \sum_{k=1}^{m-1}\left(\begin{array}{c}
2 m \\
2 k
\end{array}\right) \sum_{d \mid p}(-1)^{d-1} E_{2 k}(d+1) \widetilde{\sigma}_{2 m-2 k}(q) \\
& =-\frac{1}{8} \sum_{\substack{d\left|p \\
d^{\prime}\right| q}}(-1)^{d+d^{\prime}}\left\{E_{2 m}\left(d+d^{\prime}+1\right)+E_{2 m}\left(d-d^{\prime}+1\right)\right\} \\
& +\frac{1}{4}\left\{\widetilde{\sigma}_{0}(q) \sum_{d \mid p}(-1)^{d-1} E_{2 m}(d+1)+\widetilde{\sigma}_{2 m}(q) \widetilde{\sigma}_{0}(p)\right\}, \\
& D_{16}:=-\frac{1}{2} \sum_{k=1}^{m-1}\left(\begin{array}{c}
2 m \\
2 k
\end{array}\right) \sum_{d \mid p}(-1)^{d-1} q E_{2 k}(d+1) \widetilde{\sigma}_{2 m-2 k-1}(q)
\end{aligned}
$$




$$
\begin{aligned}
= & -\frac{q}{4} \sum_{\substack{d\left|p \\
d^{\prime}\right| q}}(-1)^{d+d^{\prime}} \frac{1}{d^{\prime}}\left\{E_{2 m}\left(d+d^{\prime}+1\right)+E_{2 m}\left(d-d^{\prime}+1\right)\right\} \\
& +\frac{q}{2}\left\{\sigma_{-1}(q) \sum_{d \mid p}(-1)^{d-1} E_{2 m}(d+1)+\widetilde{\sigma}_{2 m-1}(q) \widetilde{\sigma}_{0}(p)\right\} .
\end{aligned}
$$

Summing $D_{i}(i=1, \ldots, 16)$, and then use (2.20), we derive the theorem.

\section{References}

[1] A. Bazsó, Á. Pintér, and H. M. Srivastava, A refinement of Faulhaber's theorem concerning sums of powers of natural numbers, Appl. Math. Lett. 25 (2012), no. 3, 486-489.

[2] B. C. Berndt, Ramanujan's Notebooks. Part II, Springer-Verlag, New York, 1989.

[3] M. Besge, Extrait d'une lettre de M. Besge à M. Liouville, J. Math. Pures Appl. 7 (1862), 256

[4] B. Cho, D. Kim, and H. Park, Evaluation of a certain combinatorial convolution sum in higher level cases, J. Math. Anal. Appl. 406 (2013), no. 1, 203-210.

[5] W. Chu and R. R. Zhou, Convolutions of Bernoulli and Euler polynomials, Sarajevo J. Math. 6(19) (2010), no. 2, 147-163.

[6] A. Erdélyi, Higher Transcendental Functions. Vol 1, McGraw Hill, New York, 1953.

[7] J. W. L. Glaisher, On the square of the series in which the coefficients are the sums of the divisors of the exponents, Messenger Math. 14 (1885), 156-163.

[8] H. Hahn, Convolution sums of some functions on divisors, Rocky Mountain J. Math. 37 (2007), no. 5, 1593-1622.

[9] J. G. Huard, Z. M. Ou, B. K. Spearman, and K. S. Williams, Elementary evaluation of certain convolution sums involving divisor functions, Number theory for the millennium, II (Urbana, IL, 2000), 229-274, A K Peters, Natick, MA, 2002.

[10] D. Kim and A. Bayad, Convolution identities for twisted Eisenstein series and twisted divisor functions, Fixed Point Theory Appl. 2013 (2013), 81, 23 pp.

[11] D. Kim and N. Y. Ikikardes, Certain combinatoric Bernoulli polynomials and convolution sums of divisor functions, Adv. Difference Equ. 2013 (2013), 310, 11 pp.

[12] A. Kim, D. Kim, and L. Yan, Convolution sums arising from divisor functions, J. Korean Math. Soc. 50 (2013), no. 2, 331-360.

[13] D. Kim and Y. K. Park, Bernoulli identities and combinatoric convolution sums with odd divisor functions, Abstr. Appl. Anal. 2014 (2014), Article ID 890973, 8 pages.

[14] _ Certain Combinatoric convolution sums involving divisor functions product formulae, Taiwanese J. Math. 18 (2014), no. 3, 973-988.

[15] D. B. Lahiri, On Ramanujan's function $\tau(n)$ and the divisor function $\sigma(n)$. I, Bull. Calcutta Math. Soc. 38 (1946), 193-206.

[16] G. Melfi, On some modular identities, Number Theory (Eger, 1996), 371-382, de Gruyter, Berlin, 1998.

[17] L. Navas, F. J. Ruiz, and J. L. Varona, Old and new identities for Bernoulli polynomials via Fourier series, Int. J. Math. Math. Sci. 2012 (2012), Article ID 129126, 14 pp.

[18] S. Ramanujan, On certain arithmetical functions, Trans. Cambridge Philos. Soc. 22 (1916), no. 9, 159-184.

[19] Y. Simsek, Elliptic analogue of the Hardy sums related to elliptic Bernoulli functions, Gen. Math. 15 (2007), no. 3, 3-23.

[20] H. M. Srivastava and A. Pinter, Remarks on some relationships between the Bernoulli and Euler polynomials, Appl. Math. Lett. 17 (2004), no. 4, 375-380.

[21] Z.-H. Sun, Congruences concerning Bernoulli numbers and Bernoulli polynomials, Discrete Appl. Math. 105 (2000), no. 1-3, 193-223. 
[22] - Legendre polynomials and supercongruences, Acta Arith. 159 (2013), no. 2, $169-200$

[23] Congruences concerning Legendre polynomials. III, Int. J. Number Theory 9 (2013), no. 4, 965-999.

[24] K. S. Williams, The convolution sum $\sum_{m<n / 8} \sigma(m) \sigma(n-8 m)$, Pacific J. Math. 228 (2006), no. 2, 387-396

[25] , Number Theory in the Spirit of Liouville, London Mathematical Society, Student Texts 76, Cambridge, 2011.

[26] — The parents of Jacobi's four squares theorem are unique, Amer. Math. Monthly 120 (2013), no. 4, 329-345.

DaEyeoul Kim

National Institute for Mathematical Sciences

DAEJEON 305-811, KOREA

E-mail address: daeyeoul@nims.re.kr

ABDelmejid BAyAd

UnIVERsité D'EVRY VAL D'EsSONNE

DÉPARTEMENT DE MATHÉMATIQUES

BÂtiment I.B.G.B.I., 3Ème ÉTAGE

23 Boulevard de France

91037 Evry CEDEx, France

E-mail address: abayad@maths.univ-evry.fr

NAZLi Yildiz IKIKARdes

Department of Elementary Mathematics Education

Necatibey Faculty of Education

BALIKESIR UNIVERSITY

10100 Balikesir, Turkey

E-mail address: nyildiz@balikesir.edu.tr 